\title{
CHARACTERIZATION AND REGULARITY FOR AXISYMMETRIC SOLENOIDAL VECTOR FIELDS WITH APPLICATION TO NAVIER-STOKES EQUATION
}

By

\author{
Jian-Guo Liu \\ and
}

Wei-Cheng Wang

IMA Preprint Series \# 2227

(November 2008)

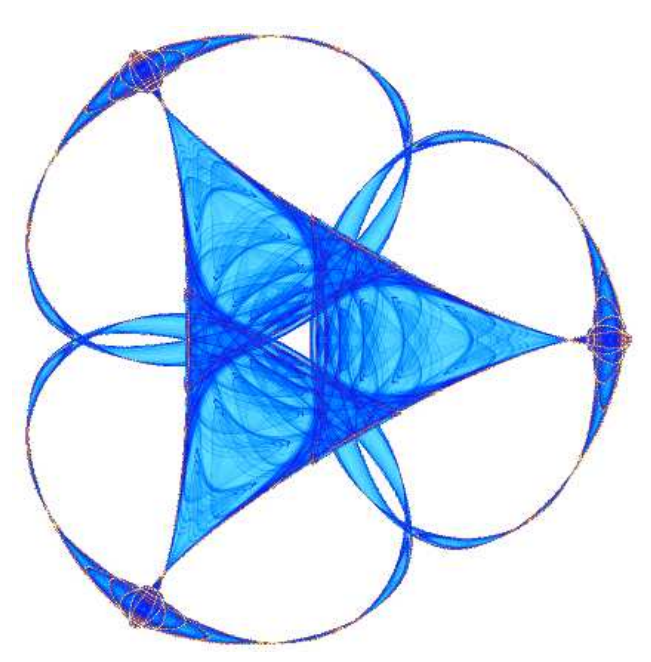

INSTITUTE FOR MATHEMATICS AND ITS APPLICATIONS

UNIVERSITY OF MINNESOTA 400 Lind Hall

207 Church Street S.E.

Minneapolis, Minnesota 55455-0436

Phone: 612-624-6066 Fax: 612-626-7370

URL: http://www.ima.umn.edu 


\title{
Characterization and Regularity for Axisymmetric Solenoidal Vector Fields with Application to Navier-Stokes Equation
}

\author{
Jian-Guo Liu ${ }^{1}$ \\ Institute for Physical Science and Technology and \\ Department of Mathematics \\ University of Maryland \\ College Park, MD 20742 \\ USA \\ and \\ Wei-Cheng Wang ${ }^{2}$ \\ Department of Mathematics \\ National Tsing Hua University \\ HsinChu, Taiwan 300 \\ TAIWAN
}

\section{Running head:}

Key words: Axisymmetric Flow, Navier Stokes, Euler Equation, Pole Condition, Pole Singularity, Leray Solution.

\section{AMS subject classifications:}

\footnotetext{
${ }^{1}$ E-mail: jliu@math.umd.edu

${ }^{2}$ E-mail: wangwc@math.nthu.edu.tw
} 


\begin{abstract}
We consider the vorticity-stream formulation of axisymmetric incompressible flows and its equivalence with the primitive formulation. It is shown that, to characterize the regularity of a divergence free axisymmetric vector field in terms of the swirling components, an extra set of pole condition is necessary to give a full description of the regularity. In addition, smooth solutions up to the axis of rotation gives rise to smooth solutions of primitive formulation in the case of Navier-Stokes equations, but not the Euler equations. We also establish proper weak formulations and show its equivalence to Leray's solutions.
\end{abstract}

\title{
1 Introduction
}

Axisymmetric flow is an important subject in fluid dynamics and has become standard textbook material as a starting point of theoretical study for complicated flow patterns. By means of Stoke's stream function $\phi[1]$, an axisymmetric divergence free vector field can be efficiently represented by two scalar components:

$$
\boldsymbol{u}=\frac{\partial_{r} \phi}{r} \boldsymbol{e}_{x}-\frac{\partial_{x} \phi}{r} \boldsymbol{e}_{r}+u \boldsymbol{e}_{\theta}
$$

Taking the swirling component of the Navier-Stokes equation

$$
\begin{aligned}
& \partial_{t} \boldsymbol{u}+(\nabla \times \boldsymbol{u}) \times \boldsymbol{u}+\nabla p=-\nu \nabla \times \nabla \times \boldsymbol{u} \\
& \nabla \cdot \boldsymbol{u}=0
\end{aligned}
$$

and the swirling component of the curl of (1.2), one can eliminate the pressure term to get two scalar convection diffusion equations:

$$
\begin{aligned}
& \partial_{t} u+u_{x} \partial_{x} u+u_{r} \partial_{r} u+\frac{u_{r}}{r} u=\nu \mathscr{L} u, \\
& \partial_{t} \omega+u_{x} \partial_{x} \omega+u_{r} \partial_{r} \omega-\frac{u_{r}}{r} \omega=\frac{1}{r} \partial_{x}\left(u^{2}\right)+\nu \mathscr{L} \omega
\end{aligned}
$$

The system is closed by the vorticity-stream function relation $\omega=-\mathscr{L} \psi, u_{x}=\frac{\partial(r \psi)}{r}$, and $u_{r}=-\partial_{x} \psi$. Here $\psi=\frac{\phi}{r}$ and $\mathscr{L} u=\partial_{r}^{2} u+\frac{\partial_{r} u}{r}+\partial_{x}^{2} u-\frac{u}{r^{2}}$.

This representation (1.3) has several advantages over the primitive formulation (1.2). It needs only two dependent variables $\psi$ and $u$ defined on $(x, r) \in\left(R \times R^{+}\right)$, and it is free 
from Lagrangian multipliers and is automatically divergence free. These advantages are particularly favorable in numerical computations.

A natural question is whether (1.3) is actually equivalent to the primitive formulation (1.2), and in which solution classes are they equivalent? In this paper, we have systematically investigated this issue for both classical and weak solutions. We start in section 2 with the characterization of smoothness of axisymmetric divergence free vector fields. It is shown that, an additional pole condition of the form

$$
\partial_{r}^{j} u\left(x, 0^{+}\right)=0, \quad \partial_{r}^{j} \psi\left(x, 0^{+}\right)=0 \quad \text { for } j \text { even }
$$

is essential to characterize the smoothness of the vector field (1.1) in classical spaces (see Lemma 2 for details). The construction of Sobolev spaces and the counter part of (1.4) are established in 2.2. We then apply this pole condition to derive regularity and equivalence results in various solution spaces in section 3. Firstly, we show in section 3.1 that there exists $C^{k}\left(R \times \overline{R^{+}}\right)$solutions of the Euler equation with a genuine singularity on the axis of rotation. In addition, this pole singularity will persist in time. In contrast, we show in section 3.2 that if the solution to (1.3) is in $C^{k}\left(R \times \overline{R^{+}}\right)$, then the pole condition (1.4) is automatically satisfied. Next, we consider weak formulation of (1.3) and study its relation with the Leray's weak solution in section 3.3. We end this paper by showing that, when appropriately formulated, the weak solutions to (1.3) are exactly the axisymmetric weak solutions obtained via Leray's construction [11].

\section{Function Spaces for Axisymmetric Solenoidal Vector Fields}

\subsection{Classical Spaces and the Pole Condition}

In this section, we establish basic regularity results for axisymmetric vector fields. We will show that the swirling component of a smooth axisymmetric vector field has vanishing even order derivatives in the radial direction at the axis of rotation. This is done in Lemma 2 by a symmetry argument.

Throughout this paper, we will be using the cylindrical coordinate system

$$
x=x, \quad y=r \cos \theta, \quad z=r \sin \theta .
$$


where the $x$-axis is the axis of rotation. A vector field $\boldsymbol{u}$ is said to be axisymmetric if $\partial_{\theta} u_{x}=\partial_{\theta} u_{r}=\partial_{\theta} u_{\theta}=0$. Here and throughout this paper, the subscripts of $u$ are used to denote components rather than partial derivatives.

The three basic differential operators in cylindrical coordinate system are given by

$$
\begin{array}{r}
\nabla u=\left(\partial_{x} u\right) \boldsymbol{e}_{x}+\left(\partial_{r} u\right) \boldsymbol{e}_{r}+\left(\frac{1}{r} \partial_{\theta} u\right) \boldsymbol{e}_{\theta} \\
\nabla \cdot \boldsymbol{u}=\frac{1}{r}\left(\partial_{x}\left(r u_{x}\right)+\partial_{r}\left(r u_{r}\right)+\partial_{\theta} u_{\theta}\right) \\
\nabla \times \boldsymbol{u}=\frac{1}{r}\left|\begin{array}{ccc}
\boldsymbol{e}_{x} & \boldsymbol{e}_{r} & r \boldsymbol{e}_{\theta} \\
\partial_{x} & \partial_{r} & \partial_{\theta} \\
u_{x} & u_{r} & r u_{\theta}
\end{array}\right|
\end{array}
$$

Here $\boldsymbol{e}_{x}, \boldsymbol{e}_{r}$ and $\boldsymbol{e}_{\theta}$ are the unit vectors in the $x, r$ and $\theta$ directions respectively.

Lemma 1 Let $\boldsymbol{u}=u_{x} \boldsymbol{e}_{x}+u_{r} \boldsymbol{e}_{r}+u_{\theta} \boldsymbol{e}_{\theta} \in C^{k}\left(R^{3}, R^{3}\right), k \geq 0$, then for any fixed $\theta \in[0, \pi)$, $u_{x}(\cdot, \cdot, \theta), u_{r}(\cdot, \cdot, \theta), u_{\theta}(\cdot, \cdot, \theta) \in C^{k}\left(R \times \overline{R^{+}}\right)$. Moreover

$$
\begin{array}{cc}
\partial_{r}^{j} u_{x}\left(x, 0^{+}, \theta\right)=(-1)^{j} \partial_{r}^{j} u_{x}\left(x, 0^{+}, \theta+\pi\right), & 0 \leq j \leq k, \\
\partial_{r}^{j} u_{r}\left(x, 0^{+}, \theta\right)=(-1)^{j+1} \partial_{r}^{j} u_{r}\left(x, 0^{+}, \theta+\pi\right), & 0 \leq j \leq k, \\
\partial_{r}^{j} u_{\theta}\left(x, 0^{+}, \theta\right)=(-1)^{j+1} \partial_{r}^{j} u_{\theta}\left(x, 0^{+}, \theta+\pi\right), & 0 \leq j \leq k .
\end{array}
$$

Proof: Let $\boldsymbol{u}=u_{x}(x, r, \theta) \boldsymbol{e}_{x}+u_{r}(x, r, \theta) \boldsymbol{e}_{r}+u_{\theta}(x, r, \theta) \boldsymbol{e}_{\theta}$. Note that $\boldsymbol{e}_{x}$ is smooth vector field while $\boldsymbol{e}_{r}$ and $\boldsymbol{e}_{\theta}$ are discontinuous at the axis of rotation. More specifically, on the cross section $z=0, y>0$, we have

$$
\begin{array}{cc}
\boldsymbol{e}_{x}(x, y, z=0)=\boldsymbol{e}_{x}(x, r=|y|, \theta=0), & \boldsymbol{e}_{x}(x,-y, z=0)=\boldsymbol{e}_{x}(x, r=|y|, \theta=\pi) \\
\boldsymbol{e}_{y}(x, y, z=0)=\boldsymbol{e}_{r}(x, r=|y|, \theta=0), & \boldsymbol{e}_{y}(x,-y, z=0)=-\boldsymbol{e}_{x}(x, r=|y|, \theta=\pi) \\
\boldsymbol{e}_{z}(x, y, z=0)=\boldsymbol{e}_{\theta}(x, r=|y|, \theta=0), & \boldsymbol{e}_{z}(x,-y, z=0)=-\boldsymbol{e}_{\theta}(x, r=|y|, \theta=\pi)
\end{array}
$$

Consequently

$$
\begin{gathered}
u_{x}(x, y, z=0)=u_{x}(x, r=|y|, \theta=0), \quad u_{x}(x,-y, z=0)=u_{x}(x, r=|y|, \theta=\pi) \\
u_{y}(x, y, z=0)=u_{r}(x, r=|y|, \theta=0), \quad u_{y}(x,-y, z=0)=-u_{x}(x, r=|y|, \theta=\pi)
\end{gathered}
$$




$$
u_{z}(x, y, z=0)=u_{\theta}(x, r=|y|, \theta=0), \quad u_{z}(x,-y, z=0)=-u_{\theta}(x, r=|y|, \theta=\pi)
$$

Taking the limit $y \rightarrow 0^{+}$, it follows that (2.5-2.7) holds with $\theta=0$. The above argument can be easily modified to prove for any other $\theta \in[0,2 \pi)$.

If $\boldsymbol{u}$ is axisymmetric, we immediately have the following direct consequence

Corollary 1 Let $\boldsymbol{u} \in C^{k}\left(R^{3}, R^{3}\right)$ be an axisymmetric vector field, $\boldsymbol{u}=u_{x}(x, r) \boldsymbol{e}_{x}+u_{r}(x, r) \boldsymbol{e}_{r}+$ $u_{\theta}(x, r) \boldsymbol{e}_{\theta}$. Then $u_{x}, u_{r}, u_{\theta} \in C^{k}\left(R \times \overline{R^{+}}\right)$and

$$
\begin{gathered}
\partial_{r}^{2 \ell+1} u_{x}\left(x, 0^{+}\right)=0, \quad 1 \leq 2 \ell+1 \leq k, \\
\partial_{r}^{2 m} u_{r}\left(x, 0^{+}\right)=\partial_{r}^{2 m} u_{\theta}\left(x, 0^{+}\right)=0, \quad 0 \leq 2 m \leq k,
\end{gathered}
$$

Denote by $\mathcal{C}_{s}^{k}$ the axisymmetric divergence free subspace of $C^{k}$ vector fields:

\section{Definition 1 :}

$$
\mathcal{C}_{s}^{k}\left(R^{3}, R^{3}\right)=\left\{\boldsymbol{u} \in C^{k}\left(R^{3}, R^{3}\right), \quad \partial_{\theta} u_{x}=\partial_{\theta} u_{r}=\partial_{\theta} u_{\theta}=0, \quad \nabla \cdot \boldsymbol{u}=0\right\}
$$

We have the following representation and regularity result for $\mathcal{C}_{s}^{k}$ :

Lemma 2 (a) For any $\boldsymbol{u} \in \mathcal{C}_{s}^{k}\left(R^{3}, R^{3}\right), k \geq 0$, there exists a unique $(u, \psi)$ such that

$$
\boldsymbol{u}=u \boldsymbol{e}_{\theta}+\nabla \times\left(\psi \boldsymbol{e}_{\theta}\right)=\frac{\partial_{r}(r \psi)}{r} \boldsymbol{e}_{x}-\partial_{x} \psi \boldsymbol{e}_{r}+u \boldsymbol{e}_{\theta}, \quad r>0,
$$

with

$$
u(x, r) \in C^{k}\left(R \times \overline{R^{+}}\right), \quad \partial_{r}^{2 \ell} u\left(x, 0^{+}\right)=0 \text { for } 0 \leq 2 \ell \leq k,
$$

and

$$
\psi(x, r) \in C^{k+1}\left(R \times \overline{R^{+}}\right), \quad \partial_{r}^{2 m} \psi\left(x, 0^{+}\right)=0 \text { for } 0 \leq 2 m \leq k+1
$$

(b) If $(u, \psi)$ satisfies (2.18), (2.19) and $\boldsymbol{u}$ is given by (2.17) for $r>0$, then $\boldsymbol{u} \in \mathcal{C}_{s}^{k}\left(R^{3}, R^{3}\right)$ with a removable singularity at $r=0$.

\section{Proof:}

$\operatorname{Part}(\mathrm{a}):$ Since $\boldsymbol{u}$ is axisymmetric, we can write $\boldsymbol{u}=u_{x}(x, r) \boldsymbol{e}_{x}+u_{r}(x, r) \boldsymbol{e}_{r}+u_{\theta}(x, r) \boldsymbol{e}_{\theta}$ for $r>0$. Rename $u_{\theta}$ by $u,(2.18)$ follows from Corollary 1. 
Next we derive the representation (2.17). Since $\boldsymbol{u}$ is divergence free, (2.3) gives

$$
\partial_{x}\left(r u_{x}\right)+\partial_{r}\left(r u_{r}\right)=0
$$

we know from standard argument that there exists a potential $\phi(x, r)$, known as Stokes' stream function, such that

$$
\partial_{x} \phi=-r u_{r}, \quad \partial_{r} \phi=r u_{x}
$$

On the cross section $z=0, y>0$, we have

$u_{x}(x, r)=u_{x}(x, y=r, z=0), \quad u_{r}(x, r)=u_{y}(x, y=r, z=0), \quad u_{\theta}(x, r)=u_{z}(x, y=r, z=0)$

From (2.20) and (2.21), it is clear that $\phi(x, r) \in C^{1}\left(R \times \overline{R^{+}}\right) \cap C^{k+1}\left(R \times R^{+}\right)$. Since $\partial_{x} \phi\left(x, 0^{+}\right)=0$, we may, without loss of generality, assume that $\phi\left(x, 0^{+}\right)=0$. This also determines $\phi$ uniquely. Next we define

$$
\psi(x, r)=\frac{\phi(x, r)}{r}, \quad r>0 .
$$

It is easy to see that $\psi(x, r) \in C^{k+1}\left(R \times R^{+}\right), \psi\left(x, 0^{+}\right)=\partial_{r} \phi\left(x, 0^{+}\right)=0$ and (2.17) follows for $r>0$.

Moreover, $\lim _{r \rightarrow 0^{+}} \partial_{r}^{j} \psi(x, r)=\lim _{r \rightarrow 0^{+}} \partial_{r}^{j} \frac{\phi(x, r)}{r}$. It follows from straight forward calculation with l'Hospital's rule and (2.20) that

$$
\partial_{r}^{j} \psi\left(x, 0^{+}\right)=\frac{j}{j+1} \partial_{r}^{j-1} u_{x}\left(x, 0^{+}\right)
$$

therefore $\psi(x, r) \in C^{k+1}\left(R \times \overline{R^{+}}\right)$. In addition, (2.19) follows from (2.14) and (2.23).

Part (b): Conversely, we now show the regularity of $\boldsymbol{u}=u \boldsymbol{e}_{\theta}+\nabla \times\left(\psi \boldsymbol{e}_{\theta}\right)$ when $(u, \psi)$ satisfies (2.18) and (2.19). Since $\boldsymbol{u}$ is axisymmetric, it suffices to check the continuity of the derivatives of $\boldsymbol{u}$ on a cross section, say $\theta=0$, or $z=0, y \geq 0$.

It is clear from $(2.17)$ and $(2.21)$ that $u_{x}(x, y, 0), u_{y}(x, y, 0)$ and $u_{z}(x, y, 0)$ have continuous $x$ derivatives up to order $k$ on $y \geq 0$. It remains to estimate the $y$-, $z$ - and mixed derivatives.

From

$$
\begin{aligned}
& \partial_{y}=\cos \theta \partial_{r}-\frac{\sin \theta}{r} \partial_{\theta} \\
& \partial_{z}=\sin \theta \partial_{r}+\frac{\cos \theta}{r} \partial_{\theta}
\end{aligned}
$$

we can derive the following 


\section{Proposition $1 \quad$ (i)}

$$
\partial_{y}^{j} F(x, r, \theta)=\cos ^{j} \theta \partial_{r}^{j} F(x, r, \theta)+\sin \theta G(x, r, \theta)
$$

where $G$ consists of the derivatives of $F$.

(ii)

$$
\begin{aligned}
\partial_{z}^{2 m}(f(x, r) \cos \theta) & =y \sum_{\ell=0}^{m} a_{\ell, m} z^{2 \ell}\left(\frac{1}{r} \partial_{r}\right)^{\ell+m}\left(\frac{f}{r}\right) \\
\partial_{z}^{2 m+1}(f(x, r) \cos \theta) & =y \sum_{\ell=0}^{m} b_{\ell, m} z^{2 \ell+1}\left(\frac{1}{r} \partial_{r}\right)^{\ell+m+1}\left(\frac{f}{r}\right) \\
\partial_{z}^{2 m}(g(x, r) \sin \theta) & =\sum_{\ell=0}^{m} c_{\ell, m} z^{2 \ell+1}\left(\frac{1}{r} \partial_{r}\right)^{\ell+m}\left(\frac{g}{r}\right) \\
\partial_{z}^{2 m-1}(g(x, r) \sin \theta) & =\sum_{\ell=0}^{m} d_{\ell, m} z^{2 \ell}\left(\frac{1}{r} \partial_{r}\right)^{\ell+m-1}\left(\frac{g}{r}\right)
\end{aligned}
$$

for some constants $a_{\ell, m}, b_{\ell, m}, c_{\ell, m}$ and $d_{\ell, m}$.

Proof: Part (i) follows straightforwardly from (2.24) and the following identity

$$
\left(\cos \theta \partial_{r}-\frac{\sin \theta}{r} \partial_{\theta}\right)\left(F_{1}+\sin \theta G_{1}\right)=\left(\cos \theta \partial_{r} F_{1}\right)+\sin \theta\left(\cos \theta \partial_{r} G_{1}-\frac{1}{r} \partial_{\theta} F_{1}-\frac{\sin \theta}{r} \partial_{\theta} G_{1}\right) .
$$

For part (ii), equations (2.27-2.30) result from substituting $\cos \theta=\frac{y}{r}, \sin \theta=\frac{z}{r}$ followed by straight forward calculations. We omit the details.

Now we proceed to show that all the mixed derivatives of orders up to $k$ are also continuous on $y \geq 0$. For simplicity of presentation, we consider mixed derivatives performed in the following order $\partial_{y}^{j} \partial_{z}^{q} \partial_{x}^{i}$. We start with $\partial_{y}^{j} \partial_{z}^{q} \partial_{x}^{i} u_{x}$ and analyze for $q$ even and odd separately.

When $q=2 m+1$, we derive from (2.25) and (2.29) that

$$
\begin{aligned}
& \partial_{y}^{j} \partial_{z}^{2 m+1} \partial_{x}^{i} u_{x}(x, y, 0) \\
= & \left.\partial_{y}^{j} \partial_{z}^{2 m}\left(\sin \theta \partial_{r} \partial_{x}^{i} u_{x}(x, r)\right)\right|_{\theta=0, r=y} \\
= & \left.\partial_{y}^{j}\left(\sum_{\ell=0}^{m} c_{\ell, m} z^{2 \ell+1}\left(\frac{1}{r} \partial_{r}\right)^{\ell+m}\left(\frac{\partial_{r} \partial_{x}^{i} u_{x}(x, r)}{r}\right)\right)\right|_{z=0, r=y} \\
= & 0
\end{aligned}
$$


Next, when $q=2 m$, it follows from (2.25), (2.26), (2.30), (2.20) and (2.22) that

$$
\begin{aligned}
& \partial_{y}^{j} \partial_{z}^{2 m} \partial_{x}^{i} u_{x}(x, y, 0) \\
= & \left.\partial_{y}^{j} \partial_{z}^{2 m-1}\left(\sin \theta \partial_{r} \partial_{x}^{i} u_{x}(x, r)\right)\right|_{\theta=0, r=y} \\
= & \left.\left(\partial_{r}^{j} \partial_{z}^{2 m-1}\left(\sin \theta \partial_{r} \partial_{x}^{i} u_{x}\right)+\sin \theta G\right)\right|_{\theta=0, r=y} \\
= & \left.\partial_{r}^{j} \sum_{\ell=0}^{m} d_{\ell, m}(r \sin \theta)^{2 \ell}\left(\frac{1}{r} \partial_{r}\right)^{\ell+m-1}\left(\frac{\partial_{r} \partial_{x}^{i} u_{x}(x, r)}{r}\right)\right|_{\theta=0, r=y} \\
= & \left.d_{0, m} \partial_{r}^{j}\left(\frac{1}{r} \partial_{r}\right)^{m} \partial_{x}^{i} u_{x}(x, r)\right|_{r=y} \\
= & \left.d_{0, m} \partial_{r}^{j}\left(\frac{1}{r} \partial_{r}\right)^{m+1}\left(r \partial_{x}^{i} \psi(x, r)\right)\right|_{r=y} .
\end{aligned}
$$

From Lemma 2 and Taylor's Theorem, we have

$$
\psi(x, r)=a_{1}(x) r+a_{3}(x) r^{3}+\cdots+a_{2 m-1}(x) r^{2 m-1}+R_{2 m+1}(\psi)
$$

where

$$
a_{\ell}(x)=\frac{1}{\ell !} \partial_{r}^{\ell} \psi\left(x, 0^{+}\right)
$$

and

$$
R_{2 m+1}(\psi)=\int_{0}^{r} \partial_{s}^{2 m+1} \psi(x, s) \frac{(r-s)^{2 m}}{(2 m) !} d s .
$$

From direct calculation, we have

$$
\left(\frac{1}{r} \partial_{r}\right)^{m+1}\left(r \partial_{x}^{i} \psi(x, r)\right)=\left(\frac{1}{r} \partial_{r}\right)^{m+1}\left(r \partial_{x}^{i} R_{2 m+1}(\psi)\right) .
$$

In addition, for $j \geq 1$, we can write

$$
R_{2 m+1}(\psi)=a_{2 m+1}(x) r^{2 m+1}+\cdots+a_{2 m+2 n+1}(x) r^{2 m+2 n+1}+R_{2 m+j+1}(\psi)
$$

where $n$ is the largest integer such that $2 n<j$. The remainder term $R_{2 m+j+1}$ satisfies $\partial_{r}^{\ell} R_{2 m+j+1}(\psi)\left(x, 0^{+}\right)=0, \quad 0 \leq \ell \leq 2 m+j, \quad \partial_{r}^{2 m+j+1} R_{2 m+j+1}(\psi)\left(x, 0^{+}\right)=\partial_{r}^{2 m+j+1} \psi\left(x, 0^{+}\right)$.

Thus, for $j \geq 0$, we have

$$
\partial_{r}^{j}\left(\frac{1}{r} \partial_{r}\right)^{m+1}\left(r \partial_{x}^{i} \psi(x, r)\right)=\partial_{r}^{j}\left(\frac{1}{r} \partial_{r}\right)^{m+1}\left(r \partial_{x}^{i} R_{2 m+j+1}(\psi)\right)=\sum_{\ell=0}^{m+j+1} C_{\ell, m} \frac{\partial_{r}^{\ell} \partial_{x}^{i} R_{2 m+j+1}(\psi)}{r^{2 m+1-\ell+j}}
$$


for some constants $C_{\ell, m}$.

From (2.34), (2.35) and l'Hospital's rule we conclude that

$$
\partial_{r}^{j}\left(\frac{1}{r} \partial_{r}\right)^{m+1}\left(r \partial_{x}^{i} \psi\right)\left(x, 0^{+}\right)=\left(\sum_{\ell=0}^{m+1} \sum_{p=0}^{j} \frac{C_{\ell, m}}{(2 m+1-\ell+j-p) !}\right) \partial_{r}^{2 m+1+j} \partial_{x}^{i} \psi\left(x, 0^{+}\right) .
$$

Since $\psi \in C^{k+1}\left(R \times \overline{R^{+}}\right)$, it follows from (2.33), (2.36) and (2.32) that $\partial_{y}^{j} \partial_{z}^{q} \partial_{x}^{i} u_{x}(x, y, 0)$ is continuous and bounded up to $y=0^{+}$for $j+q+i \leq k$.

Next we consider the mixed derivatives of $u_{y}$ and $u_{z}$. It suffices to calculate $\partial_{y}^{j} \partial_{z}^{q} \partial_{x}^{i}(f(x, r) \cos \theta+$ $g(x, r) \sin \theta)\left.\right|_{\theta=0, r=y}$ where $f$ and $g$ are either $\pm \partial_{x} \psi$ or $\pm u$.

When $q=2 m$, it follows from (2.27) and (2.29) that

$$
\begin{aligned}
& \left.\partial_{y}^{j} \partial_{z}^{2 m} \partial_{x}^{i}(f(x, r) \cos \theta+g(x, r) \sin \theta)\right|_{\theta=0, r=y} \\
= & \left.\partial_{y}^{j} \partial_{z}^{2 m}\left(\partial_{x}^{i} f(x, r) \cos \theta+\partial_{x}^{i} g(x, r) \sin \theta\right)\right|_{\theta=0, r=y} \\
= & \left.a_{0, m} \partial_{r}^{j}\left(r\left(\frac{1}{r} \partial_{r}\right)^{m}\left(\frac{\partial_{x}^{i} f}{r}\right)\right)\right|_{r=y}
\end{aligned}
$$

From (2.18-2.19), both $-\partial_{x} \psi(x, r)$ and $u(x, r)$ have local expansions of the form

$$
b_{1}(x) r+b_{3}(x) r^{3}+\cdots+b_{2 m-1}(x) r^{2 m-1}+R_{2 m+1} .
$$

Following the same argument above, we can show that both $\partial_{y}^{j} \partial_{z}^{2 m} \partial_{x}^{i} u_{y}$ and $\partial_{y}^{j} \partial_{z}^{2 m} \partial_{x}^{i} u_{z}$ are continuous and bounded up to $y=0^{+}$for $j+2 m+i \leq k$. The calculations for $\partial_{y}^{j} \partial_{z}^{2 m+1} \partial_{x}^{i} u_{y}$ and $\partial_{y}^{j} \partial_{z}^{2 m+1} \partial_{x}^{i} u_{z}$ are similar. This completes the proof of Part (b).

In view of Lemma 2, we now introduce the following function spaces:

\section{Definition 2}

$$
C_{s}^{k}\left(R \times \overline{R^{+}}\right)=\left\{f(x, r) \in C^{k}\left(R \times \overline{R^{+}}\right), \quad \partial_{r}^{2 j} f\left(x, 0^{+}\right)=0,0 \leq 2 j \leq k\right\}
$$

We can recast Lemma 2 as

Lemma 2' For $k \geq 0$,

$$
\mathcal{C}_{s}^{k}\left(R^{3}, R^{3}\right)=\left\{u \boldsymbol{e}_{\theta}+\nabla \times\left(\psi \boldsymbol{e}_{\theta}\right) \mid u \in C_{s}^{k}\left(R \times \overline{R^{+}}\right), \psi \in C_{s}^{k+1}\left(R \times \overline{R^{+}}\right)\right\}
$$

In the following sections, we will construct natural Sobolev spaces for axisymmetric divergence free vector fields, derive the counter part of Lemma 2 in these Sobolev spaces, 
and establish various regularity and equivalence results. These results rely heavily on the expression and pole condition in Lemma 2. We list below a few related Lemmas which will be used in later sections.

Lemma 3 Let $\boldsymbol{u} \in \mathcal{C}_{s}^{k}\left(R^{3}, R^{3}\right), k \geq 0$, be represented by $\boldsymbol{u}=u \boldsymbol{e}_{\theta}+\nabla \times\left(\psi \boldsymbol{e}_{\theta}\right)$ with $u \in$ $C_{s}^{k}\left(R \times \overline{R^{+}}\right)$and $\psi \in C_{s}^{k+1}\left(R \times \overline{R^{+}}\right)$. Then $(\nabla \times)^{\ell} \boldsymbol{u} \in \mathcal{C}_{s}^{k-\ell}\left(R^{3}, R^{3}\right)$ and

$$
\begin{gathered}
(\nabla \times)^{2 m} \boldsymbol{u}=(-1)^{m}\left(\left(\mathscr{L}^{m} u\right) \boldsymbol{e}_{\theta}+\nabla \times\left(\left(\mathscr{L}^{m} \psi\right) \boldsymbol{e}_{\theta}\right)\right), \quad \text { if } \quad 2 m \leq k, \\
(\nabla \times)^{2 m+1} \boldsymbol{u}=(-1)^{m+1}\left(\mathscr{L}^{m+1} \psi\right) \boldsymbol{e}_{\theta}+(-1)^{m} \nabla \times\left(\left(\mathscr{L}^{m} u\right) \boldsymbol{e}_{\theta}\right), \quad \text { if } \quad 2 m+1 \leq k,
\end{gathered}
$$

where

$$
\mathscr{L}:=\nabla^{2}-\frac{1}{r^{2}}=\left(\partial_{r}^{2}+\frac{1}{r} \partial_{r}+\partial_{x}^{2}\right)-\frac{1}{r^{2}}
$$

Moreover

$$
\begin{gathered}
\mathscr{L}^{m} u \in C_{s}^{k-2 m}\left(R \times \overline{R^{+}}\right), \quad \text { if } \quad 2 m \leq k, \\
\mathscr{L}^{m+1} \psi \in C_{s}^{k-1-2 m}\left(R \times \overline{R^{+}}\right), \quad \text { if } \quad 2 m+1 \leq k .
\end{gathered}
$$

Proof: For any $\phi \in C_{s}^{i}\left(R \times \overline{R^{+}}\right)$, we have $\phi \boldsymbol{e}_{\theta} \in \mathcal{C}_{s}^{i}$ from Lemma 2 (b). With a straight forward calculation using (2.4), it is easy to verify that for $i \geq 2$,

$$
\nabla \times \nabla \times\left(\phi \boldsymbol{e}_{\theta}\right)=-(\mathscr{L} \phi) \boldsymbol{e}_{\theta}
$$

On the other hand, it is clear that

$$
\nabla \times \nabla \times\left(\phi \boldsymbol{e}_{\theta}\right) \in \mathcal{C}_{s}^{i-2}
$$

and therefore from Lemma 2 (a),

$$
\mathscr{L} \phi \in C_{s}^{i-2}\left(R \times \overline{R^{+}}\right)
$$

The Lemma then follows from (2.38) and (2.39).

Lemma 4 If $v \in C^{k}\left(R \times \overline{R^{+}}\right)$and $v\left(x, 0^{+}\right)=0$, then

$$
\lim _{r \rightarrow 0^{+}} j \partial_{r}^{j-1}\left(\frac{v(x, r)}{r}\right)=\partial_{r}^{j} v\left(x, 0^{+}\right), \quad 1 \leq j \leq k .
$$




\section{Proof:}

Since $v \in C^{k}\left(R \times \overline{R^{+}}\right)$, we have

$$
v(x, r)=a_{1}(x) r+a_{2}(x) r^{2}+\cdots+a_{k-1}(x) r^{k-1}+R_{k}(v)
$$

from Taylor's Theorem. Here

$$
\begin{gathered}
a_{\ell}(x)=\frac{1}{\ell !} \partial_{r}^{\ell} v\left(x, 0^{+}\right), \\
R_{k}(v)=\int_{0}^{r} \partial_{r}^{k} v(x, s) \frac{(r-s)^{k-1}}{(k-1) !} d s
\end{gathered}
$$

and

$$
\partial_{r}^{\ell} R_{k}(v)\left(x, 0^{+}\right)=0, \quad 0 \leq \ell \leq k-1, \quad \partial_{r}^{k} R_{k}(v)\left(x, 0^{+}\right)=\partial_{r}^{k} v\left(x, 0^{+}\right)
$$

From (2.41), it follows that

$$
\partial_{r}^{k-1}\left(\frac{v(x, r)}{r}\right)=\partial_{r}^{k-1}\left(\frac{R_{k}(v)}{r}\right)=\sum_{\ell=0}^{k-1} C_{k-1}^{\ell}(-1)^{\ell} \ell ! \frac{\partial_{r}^{k-\ell-1} R_{k}(v)}{r^{\ell+1}}
$$

The assertion (2.40) is obvious for $j<k$. For $j=k$, from (2.42), (2.43) and l'Hospital's rule, we can easily derive

$$
\lim _{r \rightarrow 0^{+}} \partial_{r}^{k-1}\left(\frac{v(x, r)}{r}\right)=\left(\sum_{\ell=0}^{k-1} C_{k-1}^{\ell}(-1)^{\ell} \frac{1}{\ell+1}\right) \partial_{r}^{k} v\left(x, 0^{+}\right)=\frac{1}{k} \partial_{r}^{k} v\left(x, 0^{+}\right) .
$$

This completes the proof of Lemma 4.

Lemma 5 If $v \in C^{2 m}\left(R \times \overline{R^{+}}\right) \cap C_{s}^{2 m-2}\left(R \times \overline{R^{+}}\right)$then

$$
\partial_{r}^{2 m-2} \mathscr{L} v\left(\cdot, 0^{+}\right)=0 \text { if and only if } \partial_{r}^{2 m} v\left(\cdot, 0^{+}\right)=0
$$

Proof: Since

$$
\left(\nabla^{2}-\frac{1}{r^{2}}\right) v=\left(\partial_{x}^{2} v+\partial_{r}^{2} v+\partial_{r}\left(\frac{v}{r}\right)\right)
$$

one has

$$
\partial_{r}^{2 m-2} \mathscr{L} v=\left(\partial_{x}^{2} \partial_{r}^{2 m-2} v+\partial_{r}^{2 m} v+\partial_{r}^{2 m-1}\left(\frac{v}{r}\right)\right),
$$

it follows from Lemma 4 that

$$
\partial_{r}^{2 m-2} \mathscr{L} v\left(x, 0^{+}\right)=\frac{2 m+1}{2 m} \partial_{r}^{2 m} v\left(x, 0^{+}\right)
$$

and the assertion follows. 


\subsection{Sobolev Spaces}

In this section, we will construct a family of Sobolev spaces $H_{s}^{k}\left(R \times R^{+}\right)$and show a counter part for (2.37) in these Sobolev spaces: A weak solenoidal axisymmetric vector field admits the representation (2.17) with $u(x, r)$ and $\psi(x, r)$ in $H_{s}^{k}$. Moreover, both $u$ and $\psi$, together with certain even order derivatives have vanishing traces on $r=0^{+}$.

We start with the following identity for general solenoidal vector fields:

Lemma 6 If $\boldsymbol{u} \in C^{k}\left(R^{3}, R^{3}\right) \cap H^{k}\left(R^{3}, R^{3}\right)$ and $\nabla \cdot \boldsymbol{u}=0, k \geq 0$, then

$$
\|\boldsymbol{u}\|_{H^{k}\left(R^{3}, R^{3}\right)}^{2}=\sum_{\ell=0}^{k}\left\|(\nabla \times)^{\ell} \boldsymbol{u}\right\|_{L^{2}\left(R^{3}, R^{3}\right)}^{2}
$$

Proof: We prove (2.45) for $\ell$ even and odd separately.

Since $\nabla \cdot \boldsymbol{u}=0$, it follows that $\nabla \times \nabla \times \boldsymbol{u}=-\nabla^{2} \boldsymbol{u}$. Thus if $\ell$ is even, we can write

$$
\left\|(\nabla \times)^{2 m} \boldsymbol{u}\right\|_{L^{2}\left(R^{3}, R^{3}\right)}=\left\|\left(\nabla^{2}\right)^{m} \boldsymbol{u}\right\|_{L^{2}\left(R^{3}, R^{3}\right)}
$$

When $m=1$ and $u \in C^{k}\left(R^{3}\right)$, we can integrate by parts to get

$$
\int_{R^{3}}\left|\nabla^{2} u\right|^{2}=\int_{R^{3}}\left(\sum_{i_{1}=1}^{3} \partial_{i_{1}}^{2} u\right)^{2}=\int_{R^{3}} \sum_{i_{1}, i_{2}=1}^{3} \partial_{i_{1}}^{2} u \partial_{i_{2}}^{2} u=\int_{R^{3}} \sum_{i_{1}, i_{2}=1}^{3}\left(\partial_{i_{1}} \partial_{i_{2}} u\right)^{2}
$$

Similarly, when $m=2$,

$$
\begin{aligned}
\int_{R^{3}}\left|\left(\nabla^{2}\right)^{2} u\right|^{2} & =\int_{R^{3}}\left(\left(\sum_{i=1}^{3} \partial_{i}^{2}\right)^{2} u\right)^{2}=\int_{R^{3}} \sum_{i_{1}, i_{2}, i_{3}, i_{4}=1}^{3}\left(\partial_{i_{1}}^{2} \partial_{i_{2}}^{2} u\right)\left(\partial_{i_{3}}^{2} \partial_{i_{4}}^{2} u\right) \\
& =\sum_{i_{1}, i_{2}, i_{3}, i_{4}=1}^{3} \int_{R^{3}}\left(\partial_{i_{1}} \partial_{i_{2}} \partial_{i_{3}} \partial_{i_{4}} u\right)^{2} .
\end{aligned}
$$

It is therefore easy to see that

$$
\int_{R^{3}}\left|\left(\nabla^{2}\right)^{m} u\right|^{2}=\sum_{i_{1}, \cdots, i_{2 m}=1}^{3} \int_{R^{3}}\left(\partial_{i_{1}} \cdots \partial_{i_{2 m}} u\right)^{2}
$$

and consequently for $\boldsymbol{u} \in C^{k}\left(R^{3}, R^{3}\right), 2 m \leq k$,

$$
\left\|\left(\nabla^{2}\right)^{m} \boldsymbol{u}\right\|_{L^{2}\left(R^{3}, R^{3}\right)}^{2}=\sum_{i_{1}, \cdots, i_{2 m}=1}^{3}\left\|\left(\partial_{i_{1}} \cdots \partial_{i_{2 m}}\right) \boldsymbol{u}\right\|_{L^{2}\left(R^{3}, R^{3}\right)}^{2} .
$$


On the other hand, if $\ell$ is odd, we first write

$$
(\nabla \times)^{2 m+1} \boldsymbol{u}=\nabla \times\left(-\left(\nabla^{2}\right)\right)^{m} \boldsymbol{u}=(-1)^{m} \nabla \times\left(\nabla^{2}\right)^{m} \boldsymbol{u}
$$

then apply the identity

$$
\|\nabla \boldsymbol{v}\|_{L^{2}\left(R^{3}, R^{3 \times 3}\right)}^{2}=\|\nabla \times \boldsymbol{v}\|_{L^{2}\left(R^{3}, R^{3}\right)}^{2}+\|\nabla \cdot \boldsymbol{v}\|_{L^{2}\left(R^{3}\right)}^{2}
$$

to get

$$
\left\|(\nabla \times)^{2 m+1} \boldsymbol{u}\right\|_{L^{2}\left(R^{3}, R^{3}\right)}=\left\|\nabla \times\left(\nabla^{2}\right)^{m} \boldsymbol{u}\right\|_{L^{2}\left(R^{3}, R^{3}\right)}=\left\|\left(\nabla^{2}\right)^{m} \nabla \boldsymbol{u}\right\|_{L^{2}\left(R^{3}, R^{3}\right)}
$$

and from (2.47),

$$
\begin{aligned}
\left\|\left(\nabla^{2}\right)^{m} \nabla \boldsymbol{u}\right\|_{L^{2}\left(R^{3}, R^{3}\right)}^{2} & =\sum_{i, j=1}^{3}\left\|\left(\nabla^{2}\right)^{m} \partial_{i} u_{j}\right\|_{L^{2}\left(R^{3}\right)}^{2}=\sum_{i, j=1}^{3} \sum_{i_{1}, \cdots, i_{2 m}=1}^{3} \int_{R^{3}}\left(\partial_{i_{1}} \cdots \partial_{i_{2 m}} \partial_{i} u_{j}\right)^{2} \\
& =\sum_{i_{1}, \cdots, i_{2 m+1}=1}^{3}\left\|\left(\partial_{i_{1}} \cdots \partial_{i_{2 m+1}}\right) \boldsymbol{u}\right\|_{L^{2}\left(R^{3}, R^{3}\right)}^{2} .
\end{aligned}
$$

It follows from $(2.46),(2.47),(2.48)$ and (2.49) that

$$
\|\boldsymbol{u}\|_{H^{k}\left(R^{3}, R^{3}\right)}^{2}=\sum_{\ell=0}^{k} \sum_{i_{1}, \cdots, i_{\ell}=1}^{3}\left\|\partial_{i_{1}} \cdots \partial_{i_{\ell}} \boldsymbol{u}\right\|_{L^{2}\left(R^{3}, R^{3}\right)}^{2}=\sum_{\ell=0}^{k}\left\|(\nabla \times)^{\ell} \boldsymbol{u}\right\|_{L^{2}\left(R^{3}, R^{3}\right)}^{2}
$$

This completes the proof of Lemma 6 .

In Lemma 7 below, we will derive an equivalent representation of the Sobolev norms for axisymmetric solenoidal vector fields. We first introduce the following weighted Sobolev space for axisymmetric solenoidal vector fields. Let $a, b \in C^{0}\left(R \times \overline{R^{+}}\right)$, we define the weighted $L^{2}$ inner product and norm

$$
\langle a, b\rangle=\int_{-\infty}^{\infty} \int_{0}^{\infty} a(x, r) b(x, r) r d x d r, \quad\|a\|_{0}^{2}=\langle a, a\rangle,
$$

and for $a, b \in C_{s}^{1}\left(R \times \overline{R^{+}}\right)$, we define the weighted $H^{1}$ inner product and norm

$$
[a, b]=\left\langle\partial_{x} a, \partial_{x} b\right\rangle+\left\langle\partial_{r} a, \partial_{r} b\right\rangle+\left\langle\frac{a}{r}, \frac{b}{r}\right\rangle, \quad|a|_{1}^{2}=[a, a]
$$

and we define

$$
\|a\|_{1}^{2}=\|a\|_{0}^{2}+|a|_{1}^{2} .
$$


When $a \in C_{s}^{1}\left(R \times \overline{R^{+}}\right)$and $b \in C_{s}^{1}\left(R \times \overline{R^{+}}\right) \cap C^{2}\left(R \times R^{+}\right)$, we also have the following identity from integration by parts:

$$
\langle a, \mathscr{L} b\rangle=-[a, b]
$$

If $\boldsymbol{u}=u \boldsymbol{e}_{\theta}+\nabla \times\left(\psi \boldsymbol{e}_{\theta}\right)$, with $u \in C^{0}\left(R \times \overline{R^{+}}\right)$and $\psi \in C_{s}^{1}\left(R \times \overline{R^{+}}\right)$, it is easy to see that

$$
\|\boldsymbol{u}\|_{L^{2}\left(R^{3}, R^{3}\right)}^{2}=\|u\|_{0}^{2}+|\psi|_{1}^{2}
$$

Higher order Sobolev norms can be defined similarly in terms of $u$ and $\psi$ :

Definition 3 For $a \in C_{s}^{k}\left(R \times \overline{R^{+}}\right)$and $\boldsymbol{u}=u \boldsymbol{e}_{\theta}+\nabla \times\left(\psi \boldsymbol{e}_{\theta}\right) \in \mathcal{C}_{s}^{k}\left(R^{3}, R^{3}\right)$, we define

$$
\begin{aligned}
\|a\|_{H_{s}^{2 m}\left(R \times R^{+}\right)}^{2} & :=\sum_{\ell=0}^{m-1}\left\|\mathscr{L}^{\ell} a\right\|_{1}^{2}+\left\|\mathscr{L}^{m} a\right\|_{0}^{2}, & 2 m \leq k \\
\|a\|_{H_{s}^{2 m+1}\left(R \times R^{+}\right)}^{2} & :=\sum_{\ell=0}^{m}\left\|\mathscr{L}^{\ell} a\right\|_{1}^{2}, & 2 m+1 \leq k \\
\|\boldsymbol{u}\|_{\mathcal{H}_{s}^{2 m}\left(R \times R^{+}, R^{3}\right)}^{2} & :=|\psi|_{1}^{2}+\sum_{\ell=0}^{m-1}\left\|\mathscr{L}^{\ell} u\right\|_{1}^{2}+\sum_{\ell=1}^{m}\left\|\mathscr{L}^{\ell} \psi\right\|_{1}^{2}+\left\|\mathscr{L}^{m} u\right\|_{0}^{2}, & 2 m \leq k \\
\|\boldsymbol{u}\|_{\mathcal{H}_{s}^{2 m+1}\left(R \times R^{+}, R^{3}\right)}^{2} & :=|\psi|_{1}^{2}+\sum_{\ell=0}^{m}\left\|\mathscr{L}^{\ell} u\right\|_{1}^{2}+\sum_{\ell=1}^{m}\left\|\mathscr{L}^{\ell} \psi\right\|_{1}^{2}+\left\|\mathscr{L}^{m+1} \psi\right\|_{0}^{2}, & 2 m+1 \leq k
\end{aligned}
$$

When $k=0$, we denote $\|a\|_{L_{s}^{2}\left(R \times R^{+}\right)}=\|a\|_{H_{s}^{0}\left(R \times R^{+}\right)}$and $\|\boldsymbol{u}\|_{\mathcal{L}_{s}^{2}\left(R \times R^{+}, R^{3}\right)}=\|\boldsymbol{u}\|_{\mathcal{H}_{s}^{0}\left(R \times R^{+}, R^{3}\right)}$ by convention.

In view of Lemma 2, Lemma 3, Lemma 6 and (2.53), we have proved the following Lemma 7 If $\boldsymbol{u} \in \mathcal{C}_{s}^{k}\left(R^{3}, R^{3}\right), k \geq 0$, then

$$
\|\boldsymbol{u}\|_{H^{k}\left(R^{3}, R^{3}\right)}=\|\boldsymbol{u}\|_{\mathcal{H}_{s}^{k}\left(R \times R^{+}, R^{3}\right)}
$$

We can now define the Sobolev spaces for axisymmetric solenoidal vector fields following standard procedure. Denote by $C_{0}$ the space of compactly supported functions, we define

\section{Definition 4}

$$
\begin{gathered}
L_{s}^{2}\left(R \times R^{+}\right):=\text {Completion of } C_{s}^{0}\left(R \times \overline{R^{+}}\right) \cap C_{0}\left(R \times \overline{R^{+}}\right) \text {with respect to }\|\cdot\|_{0} \\
\hat{H}_{s}^{1}\left(R \times R^{+}\right):=\text {Completion of } C_{s}^{1}\left(R \times \overline{R^{+}}\right) \cap C_{0}\left(R \times \overline{R^{+}}\right) \text {with respect to }|\cdot|_{1} \\
H_{s}^{k}\left(R \times R^{+}\right):=\text {Completion of } C_{s}^{k}\left(R \times \overline{R^{+}}\right) \cap C_{0}\left(R \times \overline{R^{+}}\right) \text {with respect to }\|\cdot\|_{H_{s}^{k}\left(R \times R^{+}\right)} \\
\mathcal{H}_{s}^{k}\left(R \times R^{+}, R^{3}\right):=\text { Completion of } \mathcal{C}_{s}^{k}\left(R^{3}, R^{3}\right) \cap C_{0}\left(R^{3}, R^{3}\right) \text { with respect to }\|\cdot\|_{\mathcal{H}_{s}^{k}\left(R \times R^{+}, R^{3}\right)}
\end{gathered}
$$


With the spaces introduced above, it is easy to see that a necessary and sufficient condition for $a \in H_{s}^{k}\left(R \times R^{+}\right), k \geq 1$, is

$\mathscr{L}^{\ell} a \in H_{s}^{1}\left(R \times R^{+}\right)$, for all $0 \leq 2 \ell \leq k-1 ; \quad \mathscr{L}^{m} a \in L_{s}^{2}\left(R \times R^{+}\right)$, for all $2 \leq 2 m \leq k$.

As a consequence, we have the following characterization for the divergence free Sobolev spaces $\mathcal{H}_{s}^{k}\left(R \times R^{+}, R^{3}\right)$ :

Lemma 8 The following statements are equivalent:

1. $\boldsymbol{u} \in \mathcal{H}_{s}^{k}\left(R \times R^{+}, R^{3}\right)$

2. $\boldsymbol{u} \in H^{k}\left(R^{3}, R^{3}\right), \nabla \cdot \boldsymbol{u}=0$ and $\boldsymbol{u}$ is axisymmetric.

3. $\boldsymbol{u}=u \boldsymbol{e}_{\theta}+\nabla \times\left(\psi \boldsymbol{e}_{\theta}\right)$ with $u \in H_{s}^{k}\left(R \times R^{+}\right), \psi \in \hat{H}_{s}^{1}\left(R \times R^{+}\right)$and, if $k \geq 1$, $\mathscr{L} \psi \in H_{s}^{k-1}\left(R \times R^{+}\right)$.

When the above statements hold, we have

$$
\|\boldsymbol{u}\|_{H^{k}\left(R^{3}, R^{3}\right)}=\|\boldsymbol{u}\|_{\mathcal{H}_{s}^{k}\left(R \times R^{+}, R^{3}\right)} .
$$

Lemma 8 follows from Lemma 3, Lemma 7 and standard density argument. We omit the details.

Finally, the counterpart of (2.18) and (2.19) for $\boldsymbol{u} \in \mathcal{H}_{s}^{k}\left(R \times R^{+}, R^{3}\right)$ is given the following trace Lemma and Corollary:

Lemma 9 If $v \in \hat{H}_{s}^{1}\left(R \times R^{+}\right)$, then the trace of $v$ on $r=0$ vanishes.

Proof: For any $v \in C^{1}\left(R \times \overline{R^{+}}\right) \cap C_{0}\left(R \times \overline{R^{+}}\right)$, we have

$$
\int_{R}|v(x, 0)|^{2} d x=-2 \iint_{R \times R^{+}} v \partial_{r} v d x d r \leq \iint_{R \times R^{+}}\left(\frac{v^{2}}{r^{2}}+\left(\partial_{r} v\right)^{2}\right) r d x d r \leq\|v\|_{1}^{2}
$$

Since $v(x, 0)=0$ for $v \in C_{s}^{1}\left(R \times \overline{R^{+}}\right)$, the Lemma follows from standard density argument. Using the same density argument, we have the following

Corollary 2 (i) If $v \in H_{s}^{k}\left(R \times R^{+}\right)$, then $\mathscr{L}^{\ell} \partial_{x}^{n} v, 2 \ell+n \leq k-1$, have zero trace on $r=0$. 
(ii) If $v \in H_{s}^{k}\left(R \times R^{+}\right)$, then the trace of $\partial_{r}^{2 \ell} v$ on $r=0$ vanish for all $0 \leq 2 \ell \leq k-1$.

\section{Example 1:}

Take $\boldsymbol{u}=u \boldsymbol{e}_{\theta}$ with $u=r^{2} e^{-r}$. Note that $u=O\left(r^{2}\right)$ near the axis. Similar functions can be found in literature as initial data in numerical search for finite time singularities. Although $u \in C^{\infty}\left(R \times \overline{R^{+}}\right)$and $\boldsymbol{u}$ may appear to be a smooth vector field, it is easy to verify that $\mathscr{L} u\left(x, 0^{+}\right) \neq 0$. Thus from Lemma 2, Lemma 8 and Lemma 9, $\boldsymbol{u}$ is neither in $C^{2}\left(R^{3}, R^{3}\right)$ nor in $H^{3}\left(R^{3}, R^{3}\right)$.

\section{Axisymmetric Navier-Stokes Equations and Equiva- lence Results}

The axisymmetric Navier-Stokes equation (1.3) can be formally derived from (1.2). From Lemma 2, a smooth solution of (1.2) gives rise to a smooth solution of (1.3). However, it is not clear whether smooth solutions of (1.3) also give rise to smooth solutions of (1.2). For example, take $\nu=0$ in (1.3) and consider the Euler equation:

$$
\begin{aligned}
& \partial_{t} u+u_{x} \partial_{x} u+u_{r} \partial_{r} u+\frac{u_{r}}{r} u=0, \\
& \partial_{t} \omega+u_{x} \partial_{x} \omega+u_{r} \partial_{r} \omega-\frac{u_{r}}{r} \omega=\frac{1}{r} \partial_{x}\left(u^{2}\right), \\
& \omega=-\mathscr{L} \psi,
\end{aligned}
$$

It is easy to see that

$$
\boldsymbol{u}=u \boldsymbol{e}_{\theta}, \quad u(t, x, r)=f(r), \quad \omega=\psi \equiv 0
$$

gives rise to an exact stationary solution to (3.1) for any function $f(r) \in C^{k}\left(R \times \overline{R^{+}}\right)$, including the one given in Example 1. In other words, it is possible to have a solution in the class

$$
\begin{aligned}
& \psi(t ; x, r) \in C^{1}\left(0, T ; C^{k+1}\left(R \times \overline{R^{+}}\right)\right) \\
& u(t ; x, r) \in C^{1}\left(0, T ; C^{k}\left(R \times \overline{R^{+}}\right)\right) \\
& \omega(t ; x, r) \in C^{1}\left(0, T ; C^{k-1}\left(R \times \overline{R^{+}}\right)\right)
\end{aligned}
$$

with a genuine singularity on $r=0$ as described in Example 1. This singularity is invisible

to the $C^{k}\left(R \times \overline{R^{+}}\right)$norm. In addition, it may well persist in time. In section 3.1, we will show that the persistence of the pole singularity is indeed generic for Euler equation. 


\subsection{Propagation and Persistence of Pole Singularity}

In Euler (3.1), both $u$ and $\omega$ transport with the velocity $\left(u_{x}, u_{r}\right)=\left(\partial_{r} \psi+\frac{\psi}{r},-\partial_{x} \psi\right)$. The equation for $\psi$ is elliptic and one needs to impose one boundary condition for $\psi$. This is naturally given by

$$
\psi(x, 0)=0
$$

in view of Lemma 2. Consequently, the $r$ component of the velocity field $u_{r}=-\partial_{x} \psi$ vanishes on the boundary $r=0$ and turns it into a characteristic boundary. As a result, the value of both $u$ and $\omega$ on $r=0^{+}$are completely determined by the value of initial data on $r=0^{+}$ and the dynamics. Neither $u$ nor $\omega$ should be imposed on $r=0$. In the following Theorem, we will show that the pole singularity will propagate and remain on the boundary $r=0$. Moreover, we will show that the order of singularity will persist in time as illustrated in the special example mentioned above.

Theorem 1 Let $(\psi, u, \omega)$ be a solution to the axisymmetric Euler equation (3.1) in the class

$$
\left(\begin{array}{l}
\psi(t, x, r) \\
u(t, x, r) \\
\omega(t, x, r)
\end{array}\right) \in C^{0}\left([0, T),\left(\begin{array}{l}
C^{k+1}\left(R \times \overline{R^{+}}\right) \\
C^{k}\left(R \times \overline{R^{+}}\right) \\
C^{k-1}\left(R \times \overline{R^{+}}\right)
\end{array}\right)\right)
$$

with $k \geq 2$ and

$$
\boldsymbol{u}=\nabla \times\left(\psi \boldsymbol{e}_{\theta}\right)+u \boldsymbol{e}_{\theta}
$$

Then for $0<t<T, 0 \leq j \leq k$,

$$
\boldsymbol{u}(t, \cdot) \in \mathcal{C}_{s}^{j}\left(R^{3}, R^{3}\right) \text { if and only if } \boldsymbol{u}(0, \cdot) \in \mathcal{C}_{s}^{j}\left(R^{3}, R^{3}\right)
$$

\section{Proof:}

From 3 and (3.5), it suffices to show that, for $0<t \leq T, 0 \leq j \leq k$,

$\left\{\begin{array}{l}\partial_{r}^{2 \ell} u\left(t, \cdot, 0^{+}\right)=0, \text { for all } 2 \ell \leq j \\ \partial_{r}^{2 n} \psi\left(t, \cdot, 0^{+}\right)=0, \text { for all } 2 n \leq j+1\end{array}\right.$ if and only if $\left\{\begin{array}{l}\partial_{r}^{2 \ell} u\left(0, \cdot, 0^{+}\right)=0, \text { for all } 2 \ell \leq j \\ \partial_{r}^{2 n} \psi\left(0, \cdot, 0^{+}\right)=0, \text { for all } 2 n \leq j+1\end{array}\right.$

We will prove (3.7) by induction on $j$ using Lemma 10 below. We first prove the case $j=0$ in part (i). The induction from $j=2 m$ to $j=2 m+1$ and from $j=2 m+1$ to $j=2 m+2$ are given in part (ii) and (iii) respectively. 
Lemma 10 (i) If (3.5) holds and

$$
\psi \in C^{0}\left([0, T), C_{s}^{1}\left(R \times \overline{R^{+}}\right)\right)
$$

then for $0<t \leq T$,

$$
u(t, \cdot, \cdot) \in C_{s}^{0}\left(R \times \overline{R^{+}}\right) \quad \text { if and only if } \quad u(0, \cdot, \cdot) \in C_{s}^{0}\left(R \times \overline{R^{+}}\right)
$$

(ii) If $2 m+1 \leq k$, (3.5) holds and

$$
\psi \in C^{0}\left([0, T), C_{s}^{2 m}\left(R \times \overline{R^{+}}\right)\right), \quad u \in C^{0}\left([0, T), C_{s}^{2 m}\left(R \times \overline{R^{+}}\right)\right),
$$

then for $0<t \leq T$,

$$
\psi(t, \cdot, \cdot) \in C_{s}^{2 m+2}\left(R \times \overline{R^{+}}\right) \quad \text { if and only if } \quad \psi(0, \cdot, \cdot) \in C_{s}^{2 m+2}\left(R \times \overline{R^{+}}\right)
$$

(iii) If $2 m+2 \leq k$, (3.5) holds and

$$
\psi \in C^{0}\left([0, T), C_{s}^{2 m+2}\left(R \times \overline{R^{+}}\right)\right), \quad u \in C^{0}\left([0, T), C_{s}^{2 m}\left(R \times \overline{R^{+}}\right)\right),
$$

then for $0<t \leq T$,

$$
u(t, \cdot, \cdot) \in C_{s}^{2 m+2}\left(R \times \overline{R^{+}}\right) \quad \text { if and only if } \quad u(0, \cdot, \cdot) \in C_{s}^{2 m+2}\left(R \times \overline{R^{+}}\right)
$$

\section{Proof:}

Part (i): From the boundary condition 3.4 we know that $u_{r}\left(t, x, 0^{+}\right)=0$. From Lemma 4, we know that $\lim _{r \rightarrow 0^{+}} \frac{u_{r}}{r}=-\partial_{x r} \psi\left(t, x, 0^{+}\right)$and $u_{x}\left(t, x, 0^{+}\right)=2\left(\left.\partial_{r} \psi\right|_{r=0^{+}}\right)$. Therefore the first equation of (3.1) on $r=0^{+}$reads

$$
\partial_{t} u+2\left(\left.\partial_{r} \psi\right|_{r=0^{+}}\right) \partial_{x} u-\left(\left.\partial_{r x} \psi\right|_{r=0^{+}}\right) u=0
$$

This is a first order linear hyperbolic equation with continuous coefficients in $(t, x) \in(0, T) \times$ $R$ for $u\left(t, x, 0^{+}\right)$. Hence, for $0<t \leq T$,

$$
u\left(t, \cdot, 0^{+}\right)=0 \text { if and only if } u\left(0, \cdot, 0^{+}\right)=0
$$

Part (ii): From Lemma 5 we see that

$$
\omega \in C^{0}\left([0, T), C_{s}^{2 m-2}\left(R \times \overline{R^{+}}\right)\right),
$$


Let $v(t, x)=\partial_{r}^{2 m} \omega\left(t, x, 0^{+}\right)$, we can derive a linear hyperbolic equation for $v(t, x)$ by applying $\partial_{r}^{2 m}$ to the second equation of (3.1):

$$
\begin{gathered}
\left.\partial_{r}^{2 m}\left(u_{x} \partial_{x} \omega\right)\right|_{r=0^{+}} \\
=\left.\left.\sum_{\ell=0}^{2 m} C_{2 m}^{\ell} \partial_{r}^{\ell}\left(\partial_{r} \psi+\frac{\psi}{r}\right)\right|_{r=0^{+}} \partial_{x} \partial_{r}^{2 m-\ell} \omega\right|_{r=0^{+}} \\
=\left.\left.\sum_{\ell=0}^{2 m}\left(1+\frac{1}{\ell+1}\right) C_{2 m}^{\ell} \partial_{r}^{\ell+1} \psi\right|_{r=0^{+}} \partial_{x} \partial_{r}^{2 m-\ell} \omega\right|_{r=0^{+}} \\
\left.\partial_{r}^{2 m}\left(u_{r} \partial_{r} \omega\right)\right|_{r=0^{+}}=-\left.\left.\sum_{\ell=0}^{2 m} C_{2 m}^{\ell} \partial_{r}^{\ell}\left(\partial_{x} \psi\right)\right|_{r=0^{+}} \partial_{r} \partial_{r}^{2 m-\ell} \omega\right|_{r=0^{+}} \\
\left.\partial_{r}^{2 m}\left(\left(\partial_{x} \frac{\psi}{r}\right) \omega\right)\right|_{r=0^{+}}=\left.\left.\sum_{\ell=0}^{2 m} C_{2 m}^{\ell} \partial_{r}^{\ell}\left(\partial_{x} \frac{\psi}{r}\right)\right|_{r=0^{+}} \partial_{r}^{2 m-\ell} \omega\right|_{r=0^{+}}=\left.\left.\sum_{\ell=0}^{2 m} \frac{1}{\ell+1} C_{2 m}^{\ell} \partial_{x} \partial_{r}^{\ell+1} \psi\right|_{r=0^{+}} \partial_{r}^{2 m-\ell} \omega\right|_{r=0^{+}} \\
\left.\partial_{r}^{2 m}\left(\frac{u}{r} \partial_{x} u\right)\right|_{r=0^{+}}=\left.\left.\sum_{\ell=0}^{2 m} C_{2 m}^{\ell} \partial_{r}^{\ell}\left(\frac{u}{r}\right)\right|_{r=0^{+}} \partial_{x}\left(\partial_{r}^{2 m-\ell} u\right)\right|_{r=0^{+}}=\left.\left.\sum_{\ell=0}^{2 m} \frac{1}{\ell+1} C_{2 m}^{\ell} \partial_{r}^{\ell+1} u\right|_{r=0^{+}} \partial_{x} \partial_{r}^{2 m-\ell} u\right|_{r=0^{+}}
\end{gathered}
$$

In (3.12-3.15), we have used Lemma 4 to get

$$
\left.\partial_{r}^{\ell}\left(\frac{\psi}{r}\right)\right|_{r=0^{+}}=\left.\frac{1}{\ell+1} \partial_{r}^{\ell+1} \psi\right|_{r=0^{+}} .
$$

Next, from (3.9)

$$
\left.\partial_{r}^{2 \ell} \psi\right|_{r=0^{+}}=0,\left.\quad \partial_{r}^{2 \ell} u\right|_{r=0^{+}}=0, \quad \text { for } \ell \leq m
$$

and from (3.11)

$$
\left.\partial_{r}^{2 \ell} \omega\right|_{r=0^{+}}=0, \quad \text { for } \ell \leq m-1
$$

It follows that all the terms on the right hand side of (3.12-3.15) vanish except $\ell=0$ in $(3.12,3.14)$ and $\ell=1$ in $(3.13)$. In summary, we have

$$
\begin{gathered}
\left.\partial_{r}^{2 m}\left(u_{x} \partial_{x} \omega\right)\right|_{r=0^{+}}=2\left(\left.\partial_{r} \psi\right|_{r=0^{+}}\right) \partial_{x} v \\
\left.\partial_{r}^{2 m}\left(u_{r} \partial_{r} \omega\right)\right|_{r=0^{+}}=-2 j\left(\left.\partial_{r x} \psi\right|_{r=0^{+}}\right) v \\
\left.\partial_{r}^{2 m}\left(\left(\partial_{x} \frac{\psi}{r}\right) \omega\right)\right|_{r=0^{+}}=\left(\left.\partial_{r x} \psi\right|_{r=0^{+}}\right) v \\
\left.\partial_{r}^{2 m}\left(\frac{u}{r} \partial_{x} u\right)\right|_{r=0^{+}}=0
\end{gathered}
$$


Thus we end up with a first order hyperbolic equation with smooth coefficients for $v$ :

$$
\partial_{t} v+2\left(\left.\partial_{r} \psi\right|_{r=0^{+}}\right) \partial_{x} v-(2 m-1)\left(\left.\partial_{x r} \psi\right|_{r=0^{+}}\right) v=0
$$

It follows that for $0<t \leq T$,

$$
\partial_{r}^{2 m} \omega\left(t, \cdot, 0^{+}\right)=0 \text { if and only if } \partial_{r}^{2 m} \omega\left(0, \cdot, 0^{+}\right)=0
$$

that is, in view of Lemma 5 and (3.9),

$$
\partial_{r}^{2 m+2} \psi\left(t, \cdot, 0^{+}\right)=0 \text { if and only if } \partial_{r}^{2 m+2} \psi\left(0, \cdot, 0^{+}\right)=0
$$

for $0<t \leq T$.

Part (iii): Let $z(t, x)=\partial_{r}^{2 m+2} u\left(t, x, 0^{+}\right)$. Following a similar calculation as in part (ii), we have

$$
\begin{gathered}
\left.\partial_{r}^{2 m+2}\left(u_{x} \partial_{x} u\right)\right|_{r=0^{+}}=\left.\left.\sum_{\ell=0}^{2 m+2} C_{2 m+2}^{\ell} \partial_{r}^{\ell} u_{x}\right|_{r=0^{+}} \partial_{x}\left(\partial_{r}^{2 m+2-\ell} u\right)\right|_{r=0^{+}}=2\left(\left.\partial_{r} \psi\right|_{r=0^{+}}\right) \partial_{x} v \\
\left.\partial_{r}^{2 m+2}\left(u_{r} \partial_{r} u\right)\right|_{r=0^{+}}=\left.\left.\sum_{\ell=0}^{2 m+2} C_{2 m+2}^{\ell} \partial_{r}^{\ell} u_{r}\right|_{r=0^{+}} \partial_{x}\left(\partial_{r}^{2 m+2-\ell} u\right)\right|_{r=0^{+}}=-(2 m+2)\left(\left.\partial_{x r} \psi\right|_{r=0^{+}}\right) v
\end{gathered}
$$

and

$$
\left.\partial_{r}^{2 m+2}\left(\frac{\partial_{x} \psi}{r} u\right)\right|_{r=0^{+}}=\left.\sum_{\ell=0}^{2 m+2} C_{2 m+2}^{\ell} \partial_{r}^{\ell}\left(\frac{\partial_{x} \psi}{r}\right)\right|_{r=0^{+}} \partial_{r}^{2 m+2-\ell} u=\left(\left.\partial_{r x} \psi\right|_{r=0^{+}}\right) v
$$

We therefore obtain a first order linear hyperbolic equation with smooth coefficients for $z$ :

$$
\partial_{t} z+2\left(\left.\partial_{r} \psi\right|_{r=0^{+}}\right) \partial_{x} z-(2 m+3)\left(\left.\partial_{x r} \psi\right|_{r=0^{+}}\right) z=0
$$

Therefor, we have proved that for $0<t \leq T$,

$$
\partial_{r}^{2 m+2} u\left(t, \cdot, 0^{+}\right)=0 \text { if and only if } \partial_{r}^{2 m+2} u\left(0, \cdot, 0^{+}\right)=0
$$

This completes the proof of part (iii) and hence the proof of Theorem 1. 


\subsection{Classical Solutions of Axisymmetric Navier-Stokes Equations}

Theorem 1 reveals the subtlety of the pole singularity. In the case of Navier-Stokes equation,

$$
\begin{aligned}
& \partial_{t} u+u_{x} \partial_{x} u+u_{r} \partial_{r} u+\frac{u_{r}}{r} u=\nu \mathscr{L} u, \\
& \partial_{t} \omega+u_{x} \partial_{x} \omega+u_{r} \partial_{r} \omega-\frac{u_{r}}{r} \omega=\frac{1}{r} \partial_{x}\left(u^{2}\right)+\nu \mathscr{L} \omega \\
& \omega=-\mathscr{L} \psi,
\end{aligned}
$$

with $\nu>0$, we have an elliptic-parabolic system on a semi-bounded region $\{r>0\}$. We expect certain regularizing effect to take place. In the case the swirling velocity $u$ is zero, there exists a unique global smooth solution [10, 20]. (Result in primitive variable, translate to vorticity formulation is smooth enough) However, with the swirl velocity, whether or not initially smooth data develops singularity in finite time is is still a major open problem.A fundamental regularity result concerning the solution of the Navier-Stokes equation is given in the pioneering work of Caffarelli, Kohn and Nirenberg [3]: The one dimensional Hausdorff measure of the singular set is zero. As a consequence, the only possible singularity for axisymmetric Navier-Stokes flows would be on the axis of rotation. Further results on partial regularity for axisymmetric flow can be found in $[2,17,4,9,5]$. A recent breakthrough concerning the subtle behavior of the axisymmetric NSE can be found in [8].

Contrast to the case of Euler equation, the equivalence Theorem that we present below rules out the possibility of persistence of the pole singularity for solutions which are smooth up to the boundary $r=0$. From standard PDE theory, we need to assign boundary values for $(\psi, u, \omega)$. The zeroth order part of the pole condition $(2.18,2.19)$ would suffice:

$$
\psi(x, 0)=u(x, 0)=\omega(x, 0)=0 .
$$

It is therefore a natural question to ask if a smooth solution of $(3.27,3.28)$ in the class

$$
\begin{aligned}
& \psi(t ; x, r) \in C^{1}\left(0, T ; C^{k+1}\left(R \times \overline{R^{+}}\right)\right) \\
& u(t ; x, r) \in C^{1}\left(0, T ; C^{k}\left(R \times \overline{R^{+}}\right)\right) \\
& \omega(t ; x, r) \in C^{1}\left(0, T ; C^{k-1}\left(R \times \overline{R^{+}}\right)\right)
\end{aligned}
$$

will give rise to a smooth solution of (3.27) in the class

$$
\begin{aligned}
& \psi(t ; x, r) \in C^{1}\left(0, T ; C_{s}^{k+1}\left(R \times \overline{R^{+}}\right)\right) \\
& u(t ; x, r) \in C^{1}\left(0, T ; C_{s}^{k}\left(R \times \overline{R^{+}}\right)\right) \\
& \omega(t ; x, r) \in C^{1}\left(0, T ; C_{s}^{k-1}\left(R \times \overline{R^{+}}\right)\right)
\end{aligned}
$$


In other words, is the pole condition $(2.18,2.19)$ automatically satisfied if only the zeroth order part (3.28) is imposed?

The answer to this question is affirmative. We will show in Lemma 2 that (3.30) and (3.29) are indeed equivalent for solutions of $(3.27,3.28)$. The proof is based on local Taylor expansion. We decompose the proof into several Lemmas.

Lemma 11 If $2 m \leq k-2$ and

$$
\begin{aligned}
& \psi \in C^{k+1}\left(R \times \overline{R^{+}}\right) \cap C_{s}^{2 m}\left(R \times \overline{R^{+}}\right) \\
& u \in C^{k}\left(R \times \overline{R^{+}}\right) \cap C_{s}^{2 m}\left(R \times \overline{R^{+}}\right) \\
& \omega \in C^{k-1}\left(R \times \overline{R^{+}}\right) \cap C_{s}^{2 m}\left(R \times \overline{R^{+}}\right)
\end{aligned}
$$

then all the nonlinear terms in (3.27)

$$
u_{x} \partial_{x} \omega, \quad u_{r} \partial_{r} \omega, \quad \frac{u_{r}}{r} \omega, \quad \frac{1}{r} \partial_{x}\left(u^{2}\right)
$$

and

$$
u_{x} \partial_{x} u, \quad u_{r} \partial_{r} u, \quad \frac{u_{r}}{r} u
$$

are in $C_{s}^{2 m}\left(R \times \overline{R^{+}}\right)$.

Proof: The calculations here are analogues of the proof of Theorem 1. (3.9) holds true under the first two conditions in (3.31). hence, $\partial_{r}^{2 m}$ of four terms in (3.32) at $r=0^{+}$is given by (3.19-3.22) with $v(t, x)=\partial_{r}^{2 m} \omega\left(t, x, 0^{+}\right)$. The third condition in (3.31) implies that $v=0$. Hence, one has

$$
\left.\partial_{r}^{2 m}\left(u_{x} \partial_{x} \omega\right)\right|_{r=0^{+}}=\left.\partial_{r}^{2 m}\left(u_{r} \partial_{r} \omega\right)\right|_{r=0^{+}}=\left.\partial_{r}^{2 m}\left(\frac{u_{r}}{r} \omega\right)\right|_{r=0^{+}}=\left.\partial_{r}^{2 m}\left(\frac{1}{r} \partial_{x}\left(u^{2}\right)\right)\right|_{r=0^{+}}=0
$$

Obviously, for $j<m$, one also has

$$
\left.\partial_{r}^{2 j}\left(u_{x} \partial_{x} \omega\right)\right|_{r=0^{+}}=\left.\partial_{r}^{2 j}\left(u_{r} \partial_{r} \omega\right)\right|_{r=0^{+}}=\left.\partial_{r}^{2 j}\left(\frac{u_{r}}{r} \omega\right)\right|_{r=0^{+}}=\left.\partial_{r}^{2 j}\left(\frac{1}{r} \partial_{x}\left(u^{2}\right)\right)\right|_{r=0^{+}}=0
$$

using exactly same argument. This implies that

$$
u_{x} \partial_{x} \omega, \quad u_{r} \partial_{r} \omega, \quad \frac{u_{r}}{r} \omega, \quad \frac{1}{r} \partial_{x}\left(u^{2}\right) \in C_{s}^{2 m}\left(R \times \overline{R^{+}}\right) .
$$

Next, (3.10) holds true under the three conditions in (3.31). Hence, $\partial_{r}^{2 m}$ of three terms in (3.33) at $r=0^{+}$is given by (3.24-3.26) with $v(t, x)=\partial_{r}^{2 m} u\left(t, x, 0^{+}\right)$. The second condition 
in (3.31) implies that $v=0$. Here we used $\partial_{r}^{2 m}$ instead of $\partial_{r}^{2 m+2}$ in (3.24-3.26). Hence, one has

$$
\left.\partial_{r}^{2 m}\left(u_{x} \partial_{x} u\right)\right|_{r=0^{+}}=\left.\partial_{r}^{2 m}\left(u_{r} \partial_{r} u\right)\right|_{r=0^{+}}=\left.\partial_{r}^{2 m}\left(\frac{u_{r}}{r} u\right)\right|_{r=0^{+}}=0
$$

Again, for $j<m$, one also has

$$
\left.\partial_{r}^{2 j}\left(u_{x} \partial_{x} u\right)\right|_{r=0^{+}}=\left.\partial_{r}^{2 j}\left(u_{r} \partial_{r} u\right)\right|_{r=0^{+}}=\left.\partial_{r}^{2 j}\left(\frac{u_{r}}{r} u\right)\right|_{r=0^{+}}=0
$$

This implies that

$$
u_{x} \partial_{x} u, \quad u_{r} \partial_{r} u, \quad \frac{u_{r}}{r} u \in C_{s}^{2 m}\left(R \times \overline{R^{+}}\right) .
$$

This completes the proof of Lemma (11).

Theorem 2 If $(\psi, u, \omega)$ is a solution to (3.27, 3.28) in the class (3.29) with $k \geq 3$. Then

$$
\begin{aligned}
& \psi \in C_{s}^{k+1}\left(R \times \overline{R^{+}}\right) \\
& u \in C_{s}^{k}\left(R \times \overline{R^{+}}\right) \\
& \omega \in C_{s}^{k-1}\left(R \times \overline{R^{+}}\right)
\end{aligned}
$$

for $0<t<T$.

Proof: Let $j^{*}$ be the largest integer such that $2 j^{*} \leq k-1$. We first show that on $0<t<T$,

$$
\begin{gathered}
\partial_{r}^{2 \ell} \psi\left(t, x, 0^{+}\right)=0 \\
\partial_{r}^{2 \ell} u\left(t, x, 0^{+}\right)=0 \\
\partial_{r}^{2 \ell} \omega\left(t, x, 0^{+}\right)=0 .
\end{gathered}
$$

for $0 \leq \ell \leq j^{*}$

This is done by induction on $\ell$. When $\ell=0,(3.37)$ is given by the boundary condition (3.28). Suppose that (3.37) is verified for $\ell=j$ with $j+1 \leq j^{*}$. We apply $\left.\partial_{r}^{2 j-2}\right|_{\left(x, 0^{+}\right)}$on both sides of (3.27) and conclude that, in view of Lemma 11,

$$
\begin{gathered}
\nu \partial_{r}^{2 j}\left(\nabla^{2}-\frac{1}{r^{2}}\right) u\left(x, 0^{+}\right)=0, \\
\nu \partial_{r}^{2 j}\left(\nabla^{2}-\frac{1}{r^{2}}\right) \omega\left(x, 0^{+}\right)=0, \\
\partial_{r}^{2 j}\left(\nabla^{2}-\frac{1}{r^{2}}\right) \psi\left(x, 0^{+}\right)=0 .
\end{gathered}
$$

Apply Lemma 5 to $\partial_{r}^{2 j} \psi, \partial_{r}^{2 j} u, \partial_{r}^{2 j} \omega$, one has $\partial_{r}^{2 j+2} \psi\left(x, 0^{+}\right)=\partial_{r}^{2 j+2} u\left(x, 0^{+}\right)=\partial_{r}^{2 j+2} \omega\left(x, 0^{+}\right)=$ 0 thus (3.37) is verified for $\ell=j+1$.

We can continue the induction until (3.37) is verified for $\ell=j^{*}$ to get

$$
\begin{aligned}
& \psi \in C^{k+1}\left(R \times \overline{R^{+}}\right) \cap C_{s}^{2 j^{*}}\left(R \times \overline{R^{+}}\right) \\
& u \in C^{k}\left(R \times \overline{R^{+}}\right) \cap C_{s}^{2 j^{*}}\left(R \times \overline{R^{+}}\right) \\
& \omega \in C^{k-1}\left(R \times \overline{R^{+}}\right) \cap C_{s}^{2 j^{*}}\left(R \times \overline{R^{+}}\right)
\end{aligned}
$$


To complete the proof, we proceed with $k$ odd and even separately.

If $k$ is odd, say $k=2 m+1$, then $j^{*}=m$ and (3.38) can be written as

$$
\psi \in C^{2 m+2}\left(R \times \overline{R^{+}}\right) \cap C_{s}^{2 m}\left(R \times \overline{R^{+}}\right), \quad u \in C_{s}^{2 m+1}\left(R \times \overline{R^{+}}\right), \quad \omega \in C_{s}^{2 m}\left(R \times \overline{R^{+}}\right) .
$$

Apply Lemma 5 to $\partial_{r}^{2 m} \psi$, one has that $\partial_{r}^{2 m+2} \psi(x, 0)=0$, therefore $\psi \in C_{s}^{2 m+2}\left(R \times \overline{R^{+}}\right)$.

Similarly, if $k=2 n$, then $j^{*}=n-1$ and we have from (3.38)

$\psi \in C^{2 n+1}\left(R \times \overline{R^{+}}\right) \cap C_{s}^{2 n-2}\left(R \times \overline{R^{+}}\right), \quad u \in C^{2 n}\left(R \times \overline{R^{+}}\right) \cap C_{s}^{2 n-2}\left(R \times \overline{R^{+}}\right), \quad \omega \in C_{s}^{2 n-1}\left(R \times \overline{R^{+}}\right)$.

Since $2 n-2=k-2$, the assumption in Lemma 11 is satisfied. Therefore we can continue the induction for $u$ to get $\partial_{r}^{2 n} u\left(x, 0^{+}\right)=0$, thus $u \in C_{s}^{2 n}\left(R \times \overline{R^{+}}\right)$.

Finally, apply Lemma 5 to $\partial_{r}^{2 n-2} \psi$, we conclude that $\partial_{r}^{2 n} \psi\left(x, 0^{+}\right)=0$ and $\psi \in C^{2 n+1}(R \times$ $\left.\overline{R^{+}}\right) \cap C_{s}^{2 n}\left(R \times \overline{R^{+}}\right)=C_{s}^{2 n+1}\left(R \times \overline{R^{+}}\right)$. This completes the proof of Lemma 2.

The equivalence of (1.2) and (3.27) in terms of regularity of classical solutions is given by

Theorem 3 (I) Suppose $(\boldsymbol{u}, p)$ is an axisymmetric solution to $N S E$ (1.2) with $\boldsymbol{u} \in C^{1}\left(0, T ; \mathcal{C}_{s}^{k}\right)$, $p \in C^{0}\left(0, T ; C^{k-1}\left(R^{3}\right)\right)$ and $k \geq 3$. Then there is a solution $(\psi, u, \omega)$ to (3.27) in the class

$$
\begin{aligned}
& \psi(t, x, r) \in C^{1}\left(0, T ; C_{s}^{k+1}\left(R \times \overline{R^{+}}\right)\right) \\
& u(t, x, r) \in C^{1}\left(0, T ; C_{s}^{k}\left(R \times \overline{R^{+}}\right)\right) \\
& \omega(t, x, r) \in C^{1}\left(0, T ; C_{s}^{k-1}\left(R \times \overline{R^{+}}\right)\right)
\end{aligned}
$$

and $\boldsymbol{u}=u \boldsymbol{e}_{\theta}+\nabla \times\left(\psi \boldsymbol{e}_{\theta}\right)$.

(II) Let $(\psi, u, \omega)$ be a solution to (3.27,3.28) in the class

$$
\begin{aligned}
& \psi(t, x, r) \in C^{1}\left(0, T ; C^{k+1}\left(R \times \overline{R^{+}}\right)\right) \\
& u(t, x, r) \in C^{1}\left(0, T ; C^{k}\left(R \times \overline{R^{+}}\right)\right) \\
& \omega(t, x, r) \in C^{1}\left(0, T ; C^{k-1}\left(R \times \overline{R^{+}}\right)\right)
\end{aligned}
$$

with $k \geq 3$. Then

$$
\boldsymbol{u}=u \boldsymbol{e}_{\theta}+\nabla \times\left(\psi \boldsymbol{e}_{\theta}\right) \in C^{1}\left(0, T ; \mathcal{C}_{s}^{k}\right)
$$

and there is an axisymmetric scalar function $p \in C^{0}\left(0, T ; C^{k-1}\left(R^{3}\right)\right)$ such that $(\boldsymbol{u}, p)$ is a solution to NSE (1.2). 


\section{Proof:}

Part (I): Since $\boldsymbol{u} \in C^{1}\left(0, T ; \mathcal{C}_{s}^{k}\right)$ is a solution to (1.2) with $k \geq 3$, it follows that

$$
\boldsymbol{\omega}=\nabla \times \boldsymbol{u}=\omega \boldsymbol{e}_{\theta}+\nabla \times\left(u \boldsymbol{e}_{\theta}\right) \in C^{1}\left(0, T ; \mathcal{C}_{s}^{k-1}\right)
$$

is also an axisymmetric solution to the Navier-Stokes equation in vorticity form:

$$
\partial_{t} \boldsymbol{\omega}+\nabla \times(\boldsymbol{\omega} \times \boldsymbol{u})=-\nu \nabla \times \nabla \times \boldsymbol{\omega}
$$

Next, we express each term of (3.40) in the cylindrical coordinate as

$$
\begin{gathered}
\partial_{t} \boldsymbol{\omega}=\partial_{t} \omega \boldsymbol{e}_{\theta}+\nabla \times\left(\partial_{t} u \boldsymbol{e}_{\theta}\right), \\
-\nabla \times \nabla \times \boldsymbol{\omega}=\left(\left(\nabla^{2}-\frac{1}{r^{2}}\right) \omega\right) \boldsymbol{e}_{\theta}+\nabla \times\left(\left(\nabla^{2}-\frac{1}{r^{2}}\right) u \boldsymbol{e}_{\theta}\right),
\end{gathered}
$$

and

$$
\nabla \times(\boldsymbol{\omega} \times \boldsymbol{u})=\left(J\left(\frac{\omega}{r}, r \psi\right)-J\left(\frac{u}{r}, r u\right)\right) \boldsymbol{e}_{\theta}+\nabla \times\left(\frac{1}{r^{2}} J(r u, r \psi) \boldsymbol{e}_{\theta}\right) .
$$

From (3.41-3.43), we can rewrite (3.40) as

$$
a \boldsymbol{e}_{\theta}+\nabla \times\left(b \boldsymbol{e}_{\theta}\right)=\mathbf{0}
$$

where

$$
a=\partial_{t} \omega+J\left(\frac{\omega}{r}, r \psi\right)-J\left(\frac{u}{r}, r u\right)-\nu\left(\nabla^{2}-\frac{1}{r^{2}}\right) \omega
$$

and

$$
b=\partial_{t} u+\frac{1}{r^{2}} J(r u, r \psi)-\nu\left(\nabla^{2}-\frac{1}{r^{2}}\right) u .
$$

From (3.44), it follows that $a(x, r)=0$ and $r b(x, r)$ is a constant. Since $b\left(x, 0^{+}\right)=0$ from Lemma 11 and Lemma 2, we conclude that $b(x, r) \equiv 0$ as well. Expanding the Jacobians in above two equations we get exactly (3.27). This completes the proof of part (I).

Part (II): From Theorem 2, we know that $(\psi, u, \omega)$ satisfies (3.36). Therefore Lemma 2 applies and we have

$$
\boldsymbol{u}=u \boldsymbol{e}_{\theta}+\nabla \times\left(\psi \boldsymbol{e}_{\theta}\right) \in C^{1}\left(0, T ; \mathcal{C}_{s}^{k}\right)
$$

Next we define $\boldsymbol{\omega}=\nabla \times \boldsymbol{u}$. From (3.41-3.43), we see that $\boldsymbol{\omega}$ satisfies the Navier-Stokes equation in vorticity formulation (3.40). That is

$$
\nabla \times\left(\partial_{t} \boldsymbol{u}+\boldsymbol{\omega} \times \boldsymbol{u}+\nu \nabla \times \boldsymbol{\omega}\right)=\mathbf{0} .
$$


Thus there exists a function $p:(0, T) \rightarrow C^{k-1}\left(R^{3}\right)$ such that

$$
\partial_{t} \boldsymbol{u}+\boldsymbol{\omega} \times \boldsymbol{u}+\nu \nabla \times \boldsymbol{\omega}=-\nabla p
$$

In other words, $(\boldsymbol{u}, p)$ satisfies the NSE (1.2). Since $\boldsymbol{u} \in C^{1}\left(0, T ; \mathcal{C}_{s}^{k}\right)$, it follows from (3.45) that $\nabla p \in C^{0}\left(0, T ; \mathcal{C}_{s}^{k-2}\right)$. In addition, we can further assign $p(t)$ on a reference point $\left(x_{0}, r_{0}\right)$ so that $p \in C^{0}\left(0, T ; C^{k-1}\left(R^{3}\right)\right)$.

By construction, the left hand side of (3.45) is axisymmetric and therefore so is $\nabla p$. In particular

Therefore

$$
\partial_{\theta}\left(\nabla p \cdot \boldsymbol{e}_{\theta}\right)=\partial_{\theta}\left(\frac{1}{r} \partial_{\theta} p\right)=0 .
$$

$$
p=a(x, r) \theta+b(x, r)
$$

Since $p$ is continuous and single-valued, we conclude that $a=0$. In other words, $p$ is axisymmetric. This completes the proof of theorem.

\subsection{Weak Formulation and Leray Solution}

The Navier-Stokes equation in vorticity formulation for axisymmetric flows (3.27) can be recast as following in terms of Jacobians [14]

$$
\begin{aligned}
& u_{t}+\frac{1}{r^{2}} J(r u, r \psi)=\nu \mathscr{L} u, \\
& \omega_{t}+J\left(\frac{\omega}{r}, r \psi\right)-J\left(\frac{u}{r}, r u\right)=\nu \mathscr{L} \omega, \\
& \omega=-\mathscr{L} \psi,
\end{aligned}
$$

The expression of the nonlinear terms in (3.46) in terms of Jacobians are equivalent to the usual expression (1.3) for strong solutions. Accompanied with the Jacobians is a set of permutation identities which leads naturally to an energy and helicity preserving numerical scheme and played a key role in the convergence proof of the scheme [14, 15].

We propose the following formulation for weak solution:

Find $u \in L^{\infty}\left(0, T ; L^{2}\right) \cap L^{2}\left(0, T ; H_{s}^{1}\right), \psi \in L^{\infty}\left(0, T ; H_{s}^{1}\right)$ and $\omega \in L^{2}\left(0, T ; L^{2}\right)$ such that

$$
\begin{aligned}
& \left\langle\partial_{t} u, v\right\rangle+\left\langle\frac{v}{r^{2}}, J(r u, r \psi)\right\rangle+\nu[u, v]=0 \\
& {\left[\partial_{t} \psi, \phi\right]+\left\langle\frac{\omega}{r^{2}}, J(r \psi, r \phi)\right\rangle-\left\langle\frac{u}{r^{2}}, J(r u, r \phi)\right\rangle+\nu\langle\omega, \mathscr{L} \phi\rangle=0} \\
& \langle\omega, \xi\rangle=[\psi, \xi]
\end{aligned}
$$


for all $v \in H_{s}^{1}\left(R \times R^{+}\right), \phi \in H_{s}^{1} \cap H^{2}\left(R \times R^{+}\right)$, and $\xi \in H_{s}^{1}\left(R \times R^{+}\right)$.

Note that the viscous term in (3.47) is not treated the same way in standard variational formulation. In addition, only $u=0$ and $\psi=0$ are imposed on the boundary $r=0$. One can regard (3.47) as a variational formulation of the fourth order PDE for $\psi$ where the boundary condition $\omega=0$ is imposed implicitly. Although we have shown equivalence of NSE in vorticity-stream formulation and primitive formulation for the classic solutions which are smooth up to the boundary $r=0$. It is still not clear a priori how (3.47) is related to the weak solutions of (1.2) as constructed in Leray's seminal work [11]. To answer this question, we will show in Theorem 4 that (3.47) can be recast in standard 3D notations as:

Find $\boldsymbol{u} \in L^{\infty}\left(0, T ; L^{2}\left(R \times R^{+}, R^{3}\right)\right) \cap L^{2}\left(0, T ; \mathcal{H}_{s}^{1}\left(R \times R^{+}, R^{3}\right)\right)$ such that

$$
\left\langle\boldsymbol{v}, \partial_{t} \boldsymbol{u}+\boldsymbol{\omega} \times \boldsymbol{u}\right\rangle+\nu\langle\nabla \times \boldsymbol{v}, \nabla \times \boldsymbol{u}\rangle=0 \quad \text { for all } \boldsymbol{v} \in \mathcal{H}_{s}^{1}\left(R \times R^{+}, R^{3}\right)
$$

Now we recall Leray's definition of weak solution:

Find $\boldsymbol{u} \in L^{\infty}\left(0, T ; L^{2}\left(R^{3}, R^{3}\right)\right) \cap L^{2}\left(0, T ; H^{1}\left(R^{3}, R^{3}\right)\right)$

$$
\left\langle\boldsymbol{v}, \partial_{t} \boldsymbol{u}+\boldsymbol{\omega} \times \boldsymbol{u}\right\rangle+\nu\langle\nabla \times \boldsymbol{v}, \nabla \times \boldsymbol{u}\rangle=0 \quad \text { for all } \boldsymbol{v} \in C_{0}^{1}\left(R^{3}, R^{3}\right), \nabla \cdot \boldsymbol{v}=0
$$

Upon comparing (3.48) and (3.49), we see that the key point in establishing the equivalence result lies in a proper decomposition of a general divergence free test function into two parts, one is axisymmetric and the other has mean zero components. This is given by the following Lemma:

Lemma 12 Let $\boldsymbol{v} \in C^{1}\left(R^{3}, R^{3}\right), \nabla \cdot \boldsymbol{v}=0$, then there exists a $\boldsymbol{v}^{\text {sym }} \in \mathcal{C}_{s}^{1}\left(R^{3}, R^{3}\right)$ and

$$
\overline{v_{x}}(x, r, \theta)=v_{x}^{\text {sym }}(x, r), \quad \overline{v_{r}}(x, r, \theta)=v_{r}^{\text {sym }}(x, r), \quad \overline{v_{\theta}}(x, r, \theta)=v_{\theta}^{\text {sym }}(x, r),
$$

where

$$
\bar{f}(x, r)=\frac{1}{2 \pi} \int_{0}^{2 \pi} f(x, r, \theta) d \theta
$$

\section{Proof:}

Since $\boldsymbol{v} \in \mathcal{C}^{1}\left(R^{3}, R^{3}\right), \nabla \cdot \boldsymbol{v}=0$, there exists $\boldsymbol{\phi}=\phi_{x} \boldsymbol{e}_{x}+\phi_{r} \boldsymbol{e}_{r}+\phi_{\theta} \boldsymbol{e}_{\theta} \in C^{2}\left(R^{3}, R^{3}\right)$, such that $\nabla \times \phi=\boldsymbol{v}$. We then define

$$
\boldsymbol{v}^{\text {sym }}=\nabla \times\left(\overline{\phi_{\theta}} \boldsymbol{e}_{\theta}\right)+v_{\theta}^{s y m} \boldsymbol{e}_{\theta}, \quad v_{\theta}^{s y m}=\partial_{x} \overline{\phi_{r}}-\partial_{r} \overline{\phi_{x}} .
$$


It follows that $\boldsymbol{v}^{\text {sym }}$ is divergence free and satisfies $(3.50)$. In addition, $\phi_{x}(\cdot, \cdot, \theta), \phi_{r}(\cdot, \cdot, \theta), \phi_{\theta}(\cdot, \cdot, \theta) \in$ $C^{2}\left(R \times \overline{R^{+}}\right)$for any fixed $\theta$ in view of Corollary 1 . We therefore conclude from Bounded Convergence Theorem that

$$
\lim _{r \rightarrow 0^{+}} \frac{1}{2 \pi} \int_{0}^{2 \pi} \partial_{x}^{i} \partial_{r}^{j}\left(\begin{array}{c}
\phi_{x}(x, r, \theta) \\
\phi_{r}(x, r, \theta) \\
\phi_{\theta}(x, r, \theta)
\end{array}\right)=\frac{1}{2 \pi} \int_{0}^{2 \pi} \lim _{r \rightarrow 0^{+}} \partial_{x}^{i} \partial_{r}^{j}\left(\begin{array}{c}
\phi_{x}(x, r, \theta) \\
\phi_{r}(x, r, \theta) \\
\phi_{\theta}(x, r, \theta)
\end{array}\right), \quad 0 \leq i+j \leq 2
$$

In other words, $\overline{\phi_{x}}, \overline{\phi_{r}}, \overline{\phi_{\theta}} \in C^{2}\left(R \times \overline{R^{+}}\right)$. Moreover, $(2.14,2.15)$ imply that $\overline{\phi_{\theta}} \in C_{s}^{2}\left(R \times \overline{R^{+}}\right)$, $v_{\theta}^{\text {sym }} \in C_{s}^{1}\left(R \times \overline{R^{+}}\right)$therefore $\boldsymbol{v}^{\text {sym }} \in \mathcal{C}_{s}^{1}$.

We are now ready to show the following equivalence result.

Theorem 4 Let $\boldsymbol{u}=u \boldsymbol{e}_{\theta}+\nabla \times\left(\psi \boldsymbol{e}_{\theta}\right)$ and $\omega=\mathscr{L} \psi$. The following three statements are all equivalent.

(i) $(\psi, u, \omega)$ is a weak solution (3.47).

(ii) $\boldsymbol{u}$ is a axisymmetric weak solution defined by (3.48).

(iii) $\boldsymbol{u}$ is a Leray weak solution as defined in (3.49).

\section{Proof:}

We first show that (i) and (ii) are equivalent. Let $\boldsymbol{u}$ be an axisymmetric weak solution (3.48) and let the test function be given by $\boldsymbol{v}=v \boldsymbol{e}_{\theta}+\nabla \times\left(\phi \boldsymbol{e}_{\theta}\right)$. Simple calculation gives

$$
\begin{gathered}
\left\langle\partial_{t} \boldsymbol{u}, \boldsymbol{v}\right\rangle=\left\langle\partial_{t} u, v\right\rangle+\left[\partial_{t} \psi, \phi\right] \\
\langle\nabla \times \boldsymbol{u}, \nabla \times \boldsymbol{v}\rangle=\langle\omega, \mathscr{L} \phi\rangle+[u, v] \\
\langle\boldsymbol{\omega} \times \boldsymbol{u}, \boldsymbol{v}\rangle=\int_{R^{3}} \omega \boldsymbol{e}_{\theta} \times\left(\nabla \times\left(\psi \boldsymbol{e}_{\theta}\right)\right) \cdot\left(\nabla \times\left(\phi \boldsymbol{e}_{\theta}\right)\right)-\int_{R^{3}} u \boldsymbol{e}_{\theta} \times\left(\nabla \times\left(u \boldsymbol{e}_{\theta}\right)\right) \cdot\left(\nabla \times\left(\phi \boldsymbol{e}_{\theta}\right)\right) \\
+\int_{R^{3}} v \boldsymbol{e}_{\theta} \times\left(\nabla \times\left(u \boldsymbol{e}_{\theta}\right)\right) \cdot\left(\nabla \times\left(\psi \boldsymbol{e}_{\theta}\right)\right)
\end{gathered}
$$

In cylinder coordinates, we can write

$$
\begin{gathered}
\int_{R^{3}} a \boldsymbol{e}_{\theta} \times\left(\nabla \times\left(b \boldsymbol{e}_{\theta}\right)\right) \cdot\left(\nabla \times\left(c \boldsymbol{e}_{\theta}\right)\right) \\
=\int_{R \times R_{+}} \frac{a}{r}\left(\partial_{x}(r b) \partial_{r}(r c)-\partial_{r}(r b) \partial_{x}(r c)\right) d r d x \\
=\left\langle\frac{a}{r^{2}}, J(r b, r c)\right\rangle
\end{gathered}
$$


Hence

$$
\langle\boldsymbol{\omega} \times \boldsymbol{u}, \boldsymbol{v}\rangle=\left\langle\frac{\omega}{r^{2}}, J(r \psi, r \phi)\right\rangle-\left\langle\frac{u}{r^{2}}, J(r u, r \phi)\right\rangle+\left\langle\frac{v}{r^{2}}, J(r u, r \psi)\right\rangle
$$

Since $v$ and $\phi$ are independent, it follows from (3.52), (3.53), (3.54) and (3.48) that

$$
\begin{gathered}
\left\langle\partial_{t} u, v\right\rangle+\left\langle\frac{v}{r^{2}}, J(r u, r \psi)\right\rangle+\nu[u, v]=0 \\
{\left[\partial_{t} \psi, \phi\right]+\left\langle\frac{\omega}{r^{2}}, J(r \psi, r \phi)\right\rangle-\left\langle\frac{u}{r^{2}}, J(r u, r \phi)\right\rangle+\nu\langle\omega, \mathscr{L} \phi\rangle=0}
\end{gathered}
$$

Together weak formulation of relation $\omega=\mathscr{L} \psi$ :

$$
[\psi, \xi]=\langle\omega, \xi\rangle \quad \text { for all } \xi \in H_{s}^{1}\left(R \times R^{+}\right) \text {. }
$$

Hence $(\psi, u, \omega)$ is a weak solution to (3.47). The converse is also true by reversing the calculations above. This proves the equivalence between (i) and (ii).

Since $\mathcal{C}_{s}^{1}\left(R^{3}, R^{3}\right) \cap C_{c}\left(R^{3}, R^{3}\right)$ is a subspace of $\left\{\boldsymbol{v} \in C_{0}^{1}\left(R^{3}, R^{3}\right), \nabla \cdot \boldsymbol{v}=0\right\}$, and is dense in $\mathcal{H}_{s}^{1}\left(R \times R^{+}, R^{3}\right)$, (iii) implies (ii).

It remains to show that (ii) implies (iii). Let $\boldsymbol{u}$ be an axisymmetric weak solution of (3.48). From Lemma 12, for any test function $\boldsymbol{v} \in C_{0}^{1}\left(R^{3}, R^{3}\right)$ with $\nabla \cdot \boldsymbol{v}=0$, we can construct $\boldsymbol{v}^{\text {sym }} \in \mathcal{C}_{s}^{1}\left(R^{3}, R^{3}\right) \cap C_{0}\left(R^{3}, R^{3}\right)$ such that

$$
\int_{0}^{2 \pi}\left(\boldsymbol{v}-\boldsymbol{v}^{\text {sym }}\right)(x, r, \theta) d \theta=0, \quad \text { for all }(x, r) \in\left(R \times R^{+}\right)
$$

For any $\boldsymbol{w} \in L_{s}^{2}\left(R^{3}, R^{3}\right)$, one has

$$
\int_{0}^{2 \pi}\left(\boldsymbol{v}-\boldsymbol{v}^{\text {sym }}\right) \cdot \boldsymbol{w}(x, r, \theta) d \theta=0, \quad \text { for all }(x, r) \in\left(R \times R^{+}\right)
$$

and

$$
\int_{0}^{2 \pi} \nabla \times\left(\boldsymbol{v}-\boldsymbol{v}^{\text {sym }}\right) \cdot \boldsymbol{w}(x, r, \theta) d \theta=0, \quad \text { for all }(x, r) \in\left(R \times R^{+}\right)
$$

Hence

$$
\left\langle\boldsymbol{v}, \partial_{t} \boldsymbol{u}+\boldsymbol{\omega} \times \boldsymbol{u}\right\rangle+\nu\langle\nabla \times \boldsymbol{v}, \nabla \times \boldsymbol{u}\rangle=\left\langle\boldsymbol{v}^{\text {sym }}, \partial_{t} \boldsymbol{u}+\boldsymbol{\omega} \times \boldsymbol{u}\right\rangle+\nu\left\langle\nabla \times \boldsymbol{v}^{\text {sym }}, \nabla \times \boldsymbol{u}\right\rangle
$$

But now $\boldsymbol{v}^{\text {sym }} \in \mathcal{C}_{s}^{1}\left(R^{3}, R^{3}\right) \cap C_{0}\left(R^{3}, R^{3}\right) \subset \mathcal{H}_{s}^{1}\left(R \times R^{+}, R^{3}\right)$ is a test function for the axisymmetric weak solution (3.48), so the right hand side of (3.61) is zero. Therefore $\boldsymbol{u}$ is a Leray solution. This completes the proof of this Theorem. 
Corollary 3 (i) For any initial data $u_{0} \in L^{2}\left(R \times R^{+}\right), \psi_{0} \in \hat{H}_{s}^{1}\left(R \times R^{+}\right)$, there is a global weak solution $(\psi, u, \omega)$ to (3.47), and $\boldsymbol{u}=u \boldsymbol{e}_{\theta}+\nabla \times\left(\psi \boldsymbol{e}_{\theta}\right)$ is an axisymmetric Leray solution of the Navier-Stokes equation (1.2).

(ii) If in addition,

$$
u_{0} \in H_{s}^{k}\left(R \times R^{+}\right), \psi_{0} \in \hat{H}_{s}^{1}\left(R \times R^{+}\right), \mathscr{L} \psi_{0} \in H_{s}^{k-1}\left(R \times R^{+}\right)
$$

with $k \geq 1$, then there exists a $T_{0}>0$, such that the solution satisfies

$$
\begin{aligned}
& u \in C^{0}\left(0, T_{0} ; H_{s}^{k}\left(R \times R^{+}\right)\right) \cap L^{2}\left(0, T_{0} ; H_{s}^{k+1}\left(R \times R^{+}\right)\right) \\
& \omega \in C^{0}\left(0, T_{0} ; H_{s}^{k-1}\left(R \times R^{+}\right)\right) \cap L^{2}\left(0, T_{0} ; H_{s}^{k}\left(R \times R^{+}\right)\right)
\end{aligned}
$$

and it corresponds to the unique strong solution of Navier-Stokes equation (1.2).

(iii) If $k \geq 3$ in (3.62), then the solution is classical:

$$
\begin{aligned}
& u \in C^{0}\left(0, T_{0} ; C_{s}^{k-2}\left(R \times \overline{R^{+}}\right)\right) \cap C^{1}\left(0, T_{0} ; C^{k-3}\left(R \times \overline{R^{+}}\right)\right) \\
& \psi \in C^{0}\left(0, T_{0} ; C_{s}^{k-1}\left(R \times \overline{R^{+}}\right)\right) \cap C^{1}\left(0, T_{0} ; C^{k-2}\left(R \times \overline{R^{+}}\right)\right)
\end{aligned}
$$

Proof: From an initial data $u_{0} \in L^{2}\left(R \times R^{+}\right), \psi_{0} \in \hat{H}_{s}^{1}\left(R \times R^{+}\right)$, one can construct an axisymmetric vector field $\boldsymbol{u}_{0}=u_{0} \boldsymbol{e}_{\theta}+\nabla \times\left(\psi_{0} \boldsymbol{e}_{\theta}\right) \in L_{s}^{2}\left(R^{3}, R^{3}\right)$, and then a global weak solution of (3.49) using Leray's method with initial data $\boldsymbol{u}_{0}$. The weak solution is constructed from a family of approximate solutions obtained via (radially symmetric) mollifiers. See $[11,16]$ for details. Since the symmetry with respect to the axis of rotation is preserved under the action of convolution with the mollifiers, the resulting limit is also axisymmetric. From Theorem 4, it corresponds to a global weak solution $(\psi, u, \omega)$ of $(3.47)$. This shows part (i)

If in addition, $u_{0} \in H_{s}^{k}\left(R \times R^{+}\right), \mathscr{L} \psi_{0} \in H_{s}^{k-1}\left(R \times R^{+}\right), k \geq 1$ then $\boldsymbol{u}_{0} \in \mathcal{H}_{s}^{k}\left(R \times R^{+}, R^{3}\right)$. Hence by classical theory of Navier-Stokes equation [18], there exists a $T_{0}>0$ depending only on $\nu$ and $\left\|\boldsymbol{u}_{0}\right\|_{H^{k}\left(R^{3}, R^{3}\right)}$, and a unique solution $(\boldsymbol{u}, p)$ in $\left(0, T_{0}\right)$ to (1.2) with regularity

$$
\begin{gathered}
\boldsymbol{u} \in H^{1}\left(0, T_{0} ; H^{k-1}\left(R^{3}, R^{3}\right)\right) \cap L^{2}\left(0, T_{0} ; H^{k+1}\left(R^{3}, R^{3}\right)\right), \\
\nabla p \in L^{2}\left(0, T_{0} ; H^{k-1}\left(R^{3}, R^{3}\right)\right)
\end{gathered}
$$

From [6, p. 288], (3.65) implies

$$
\boldsymbol{u} \in C^{0}\left(0, T_{0} ; H^{k}\left(R^{3}, R^{3}\right)\right)
$$


Consequently, any global weak solution of (3.47) coincides with the strong solution (3.65) in $\left(o, T_{0}\right)$, therefore the strong solution is also axisymmetric. It follows from Lemma 8 that $u \in L^{\infty}\left(0, T_{0} ; H_{s}^{k}\left(R \times R^{+}\right)\right) \cap L^{2}\left(0, T_{0} ; H_{s}^{k+1}\left(R \times R^{+}\right)\right), \omega \in L^{\infty}\left(0, T_{0} ; H_{s}^{k-1}\left(R \times R^{+}\right)\right) \cap$ $L^{2}\left(0, T_{0} ; H_{s}^{k}\left(R \times R^{+}\right)\right)$. This shows part (ii).

Since $H^{2}\left(R^{3}, R^{3}\right) \subset C^{0}\left(R^{3}, R^{3}\right)$, it follows from (3.67) that, when $k \geq 3$,

$$
\boldsymbol{u} \in C^{0}\left(0, T_{0} ; C_{s}^{k-1}\left(R^{3}, R^{3}\right)\right)
$$

Since $\partial_{t} \boldsymbol{u}$ is the Leray projection of $\nu \nabla^{2} \boldsymbol{u}-(\nabla \times \boldsymbol{u}) \times \boldsymbol{u}$, it follows that

$$
\partial_{t} \boldsymbol{u} \in C^{0}\left(0, T_{0} ; C_{s}^{k-3}\left(R^{3}, R^{3}\right)\right)
$$

This gives (3.64) and proves (iii).

From well known regularity results of 3D Euler equation, the counter part of Corollary 3 for the Euler equation can be obtained using a similar argument. We state it without proof.

Corollary 4 For any initial data $u_{0} \in H_{s}^{k}\left(R \times R^{+}\right), \psi_{0} \in \hat{H}_{s}^{1}\left(R \times R^{+}\right), \mathscr{L} \psi_{0} \in H_{s}^{k-1}\left(R \times R^{+}\right)$, $k \geq 3$, there exists a unique local-in-time classical solution $(\psi, u, \omega)$ to the Euler equation (3.1) with

$$
\begin{aligned}
& u \in C^{0}\left(0, T_{0} ; C_{s}^{k-2}\left(R \times \overline{R^{+}}\right) \cap H_{s}^{k}\left(R \times R^{+}\right)\right) \cap C^{1}\left(0, T_{0} ; C^{k-3}\left(R \times \overline{R^{+}}\right) \cap H_{s}^{k-1}\left(R \times R^{+}\right)\right) \\
& \psi \in C^{0}\left(0, T_{0} ; C_{s}^{k-1}\left(R \times \overline{R^{+}}\right) \cap H_{s}^{k+1}\left(R \times R^{+}\right)\right) \cap C^{1}\left(0, T_{0} ; C^{k-2}\left(R \times \overline{R^{+}}\right) \cap H_{s}^{k}\left(R \times R^{+}\right)\right)
\end{aligned}
$$

As remarked earlier, the weak formulation (3.47) is not standard and it only imposes the boundary condition $\omega=0$ in an implicitly way. In fact, if the solution is regular enough, then one recovers this boundary condition and the usual weak formulation follows. This becomes more clear as we recast part (ii) of Corollary 3 as follows

Corollary 5 Let $(\psi, u, \omega)$ be a weak solution of (3.47) and $\left.\boldsymbol{u}=\nabla \times\left(\psi \boldsymbol{e}_{\theta}\right)+u \boldsymbol{e}_{\theta}\right)$. If

$$
\boldsymbol{u} \in L_{l o c}^{\infty}\left((0, T) ; \mathcal{H}_{s}^{1}\left(R \times R^{+}, R^{3}\right)\right)
$$

then

$$
\boldsymbol{u} \in L_{l o c}^{2}\left((0, T) ; \mathcal{H}_{s}^{2}\left(R \times R^{+}, R^{3}\right)\right)
$$

In particular, the trace of $\omega=\mathscr{L} \psi$ on $r=0^{+}$vanishes almost everywhere on $(0, T)$. 
Remark 1 The standard variational formulation for (1.3) is as follows:

Find $u \in L^{\infty}\left(0, T ; L^{2}\right) \cap L^{2}\left(0, T ; H_{s}^{1}\right), \psi \in L^{\infty}\left(0, T ; H_{s}^{1}\right)$ and $\omega \in L^{2}\left(0, T ; H_{s}^{1}\right)$ such that

$$
\begin{aligned}
& \left\langle\partial_{t} u, v\right\rangle+\left\langle\frac{v}{r^{2}}, J(r u, r \psi)\right\rangle+\nu[u, v]=0 \\
& {\left[\partial_{t} \psi, \phi\right]+\left\langle\frac{\omega}{r^{2}}, J(r \psi, r \phi)\right\rangle-\left\langle\frac{u}{r^{2}}, J(r u, r \phi)\right\rangle-\nu[\omega, \phi]=0} \\
& \langle\omega, \xi\rangle=[\psi, \xi]
\end{aligned}
$$

for all $v \in H_{s}^{1}\left(R \times \overline{R^{+}}\right), \phi \in H_{s}^{1}\left(R \times \overline{R^{+}}\right)$, and $\xi \in H_{s}^{1}\left(R \times \overline{R^{+}}\right)$.

The main difference between (3.47) and (3.71) is the viscous term of the vorticity equation. The formulation (3.71) is natural for standard $C^{0}$ finite element setting. The regularity requirement for (3.71) lies between weak solution (3.47) and the strong solution (3.65). The well-posedness of (3.71), including uniqueness and local existence of solution for initial data $u_{0} \in L_{s}^{2}\left(R \times R^{+}\right), \omega_{0} \in L_{s}^{2}\left(R \times R^{+}\right)$is still unclear.

\section{Acknowledgments}

J.-G. Liu is sponsored in part by NCTS and Ministry of Education of Taiwan and NSF grant DMS 05-12176 and DMS 08-11177. W.C. Wang is sponsored in part by NCTS and NSC grant 92-2115-M-007-022.

\section{References}

[1] G. K. Batchelor, An introduction to fluid dynamics, Cambridge University Press, Cambridge, 1999.

[2] J.T. Beale, T. Kato and A. Majda, Remarks on the breakdown of smooth solutions for the 3-D Euler equations, Comm. Math. Phys. 94 (1984), 61-66.

[3] L. Caffarelli, R. Kohn and L. Nirenberg, Partial regularity of suitable weak solutions of the Navier-Stokes equations, Comm. Pure Appl. Math. 35 (1982) 771-831.

[4] D. Chae and J. Lee, On the regularity of axisymmetric solutions of the Navier-Stokes equations, Math. Z. 239 (2002) 645-671. 
[5] C.C. Chen, R. M. Strain, H.-T. Yau, and T.-P. Tsai, Lower bound on the blow-up rate of the axisymmetric NavierStokes equations, International Mathematics Research Notices, (2008)

[6] L. C. Evans, Partial Differential Equations. Amer. Math. Soc., Providence, 1998.

[7] R. Grauer and T.C. Sideris, Finite time singularities in ideal fluids with swirl, Physica D 88 (1995) 116-132.

[8] T. Y. Hou and C.-M. Li, Dynamic stability of the 3D axi-symmetric Navier-Stokes equations with swirl, Comm. Pure Appl. Math. 61 (2008), 661-697.

[9] Q. Jiu and Z. Xin, Viscous approximations and decay rate of maximal vorticity function for 3-D axisymmetric Euler equations. Acta Math. Sin. (Engl. Ser.) 20 (2004), 385-404.

[10] O. Ladyzhenskaya, The Mathematical Theory of Viscous Incompressible Flow, Gordon and Breach, New York, 1969.

[11] J. Leray, Sur le mouvement dún liquide visqueux emplissant léspace, Acta Mathematica 63 (1934) 193-248,

[12] F. Lin, A new proof of the Caffarelli-Kohn-Nirenberg theorem, Comm. Pure Appl. Math., $51(1998) 241-57$.

[13] F.-H. Lin and C. Liu, Partial Regularities of the Nonlinear Dissipative Systems Modeling the Flow of Liquid Crystals, Discrete and Continuous Dynamic Systems, 2 (1996) 1-23.

[14] J.-G. Liu and W.C. Wang, Energy and Helicity Preserving Schemes for Hydro- and Magnetohydro-dynamics Flows with Symmetry, J. Comput. Phys., 200 (2004) 8-33.

[15] J.-G. Liu and W.-C. Wang, Convergence analysis for the energy and helicity preserving scheme for axisymmetric flows, SIAM J. Num. Ana., 44 (2006), 2456-2480.

[16] A. J. Majda and A. L. Bertozzi, Vorticity and Incompressible Flow, Cambridge University Press, 2002. 
[17] J. Neustupa and M. Pokorný, An interior regularity criterion for an axially symmetric suitable weak solution to the NavierStokes equations, Journal of Mathematical Fluid Mechanics 2 (2000) 381-99.

[18] R. Temam, Navier-Stokes Equations: Theory and Numerical Analysis, American Mathematical Society, 2001

[19] G. Tian, Z. Xin, Gradient estimation on Navier-Stokes equations, Communications in Analysis and Geometry 7 (1999) 221-57.

[20] M.R. Uchovskii and B.I. Yudovich, Axially symmetric flows of an ideal and viscous fluid, J. Appl. Math. Mech. 32 (1968) 52-61. 